\title{
A Comparison of Three Algorithms for Orion Drogue Parachute Release
}

\author{
Daniel A. Matz* and Robert D. Braun ${ }^{\dagger}$ \\ Georgia Institute of Technology, Atlanta, GA, 30332
}

\begin{abstract}
The Orion Multi-Purpose Crew Vehicle is susceptible to flipping apex forward between drogue parachute release and main parachute inflation. A smart drogue release algorithm is required to select a drogue release condition that will not result in an apex forward main parachute deployment. The baseline algorithm is simple and elegant, but does not perform as well as desired in drogue failure cases. A simple modification to the baseline algorithm can improve performance, but can also sometimes fail to identify a good release condition. A new algorithm employing simplified rotational dynamics and a numeric predictor to minimize a rotational energy metric is proposed. A Monte Carlo analysis of a drogue failure scenario is used to compare the performance of the algorithms. The numeric predictor prevents more of the cases from flipping apex forward, and also results in an improvement in the capsule attitude at main bag extraction. The sensitivity of the numeric predictor to aerodynamic dispersions, errors in the navigated state, and execution rate is investigated, showing little degradation in performance.
\end{abstract}

\section{Nomenclature}

\begin{tabular}{ll}
$\alpha$ & Angle of attack \\
$\alpha_{\text {hang }}$ & Angle of attack when hanging under the parachutes \\
$\alpha_{\text {total }}$ & Total angle of attack \\
$\alpha_{\text {trim }}$ & Free flight trim angle of attack \\
$\beta$ & Sideslip angle \\
$\beta_{\text {hang }}$ & Sideslip angle when hanging under the parachutes \\
$\beta_{\text {trim }}$ & Free flight trim sideslip angle \\
$c$ & Aerodynamic reference length of the MPCV \\
$\left(C_{D} S\right)_{\text {chute }}$ & Drag area of the parachute cluster \\
$C_{m_{\alpha}}$ & Derivative of the pitching moment with respect to angle of attack \\
$C_{m_{q}}$ & Derivative of the pitching moment with respect to pitch rate \\
$C_{n_{\beta}}$ & Derivative of the yawing moment with respect to sideslip angle \\
$q$ & Dynamic pressure \\
$\underline{R}$ & Position of the parachute attach point with respect to the center of gravity \\
\hline$S$ & Aerodynamic reference area of the MPCV \\
$\underline{v}$ & Freestream velocity vector \\
$\underline{\omega}$ & Angular velocity \\
BET & Best Estimated Trajectory \\
CDT & Cluster Development Test \\
CPAS & Capsule Parachute Assembly System \\
EFT & Exploration Flight Test \\
EM & Exploration Mission \\
FBC & Forward Bay Cover \\
GRAM & Global Reference Atmospheric Model \\
\end{tabular}

*Graduate Student, Guggenheim School of Aerospace Engineering, AIAA Member.

${ }^{\dagger}$ David and Andrew Lewis Professor of Space Technology, Guggenheim School of Aerospace Engineering, AIAA Fellow. 


$\begin{array}{ll}\text { MPCV } & \text { Multi-Purpose Crew Vehicle } \\ \text { PA } & \text { Pad Abort } \\ \text { RCS } & \text { Reaction Control System } \\ \text { SDR } & \text { Smart Drogue Release }\end{array}$

\section{Introduction}

$\mathrm{T}$ HE Orion Multi-Purpose Crew Vehicle (MPCV) is NASA's next generation manned spacecraft, capable of supporting a variety of exploration missions beyond low Earth orbit. The Orion vehicle utilizes a capsule geometry for re-entry, and is capable of both direct and skip entries. Reaction control system (RCS) jets control the vehicle attitude during re-entry to target a water landing off the coast of the Baja Peninsula. The aerodynamic forces acting on the vehicle decelerate it to subsonic speeds, and a parachute system is then used to further decelerate for landing. The first entry flight test, called Exploration Flight Test 1 (EFT-1), is scheduled for December 2014. The second entry flight test is designated Exploration Mission 1 (EM-1), and is scheduled for no earlier than late 2017. The first manned flight is designated EM-2.

The government furnished MPCV Capsule Parachute Assembly System (CPAS) utilizes four sets of parachutes during its nominal descent sequence. At an altitude of $24000 \mathrm{ft}$, three Forward Bay Cover (FBC) pilot parachutes are deployed. The FBC is jettisoned 1.4s later, giving the FBC pilots time to properly inflate. The FBC pilots remain attached to the FBC, pulling it through the MPCV wake and providing positive mid field separation. Two drogue parachutes are mortar deployed $2.0 \mathrm{~s}$ after FBC jettison, so as to provide time for the FBC to separate from the MPCV and prevent recontact of the FBC with the drogues. The drogues decelerate the MPCV from a Mach number of 0.6 to roughly 0.2 . The MPCV is dynamically unstable at these low Mach numbers, and so the drogues also serve the purpose of maintaining the capsule's rotational stability. Drogue release is permitted during a time and altitude window which begins at an altitude of $8000 \mathrm{ft}$. The smart drogue release (SDR) algorithm determines when during the window to command the release. At the same time the drogues are released, the main pilot parachutes are mortar deployed. These parachutes extract the main parachute bags and deploy the three main parachutes. The full parachute sequence is illustrated in Figure 1.

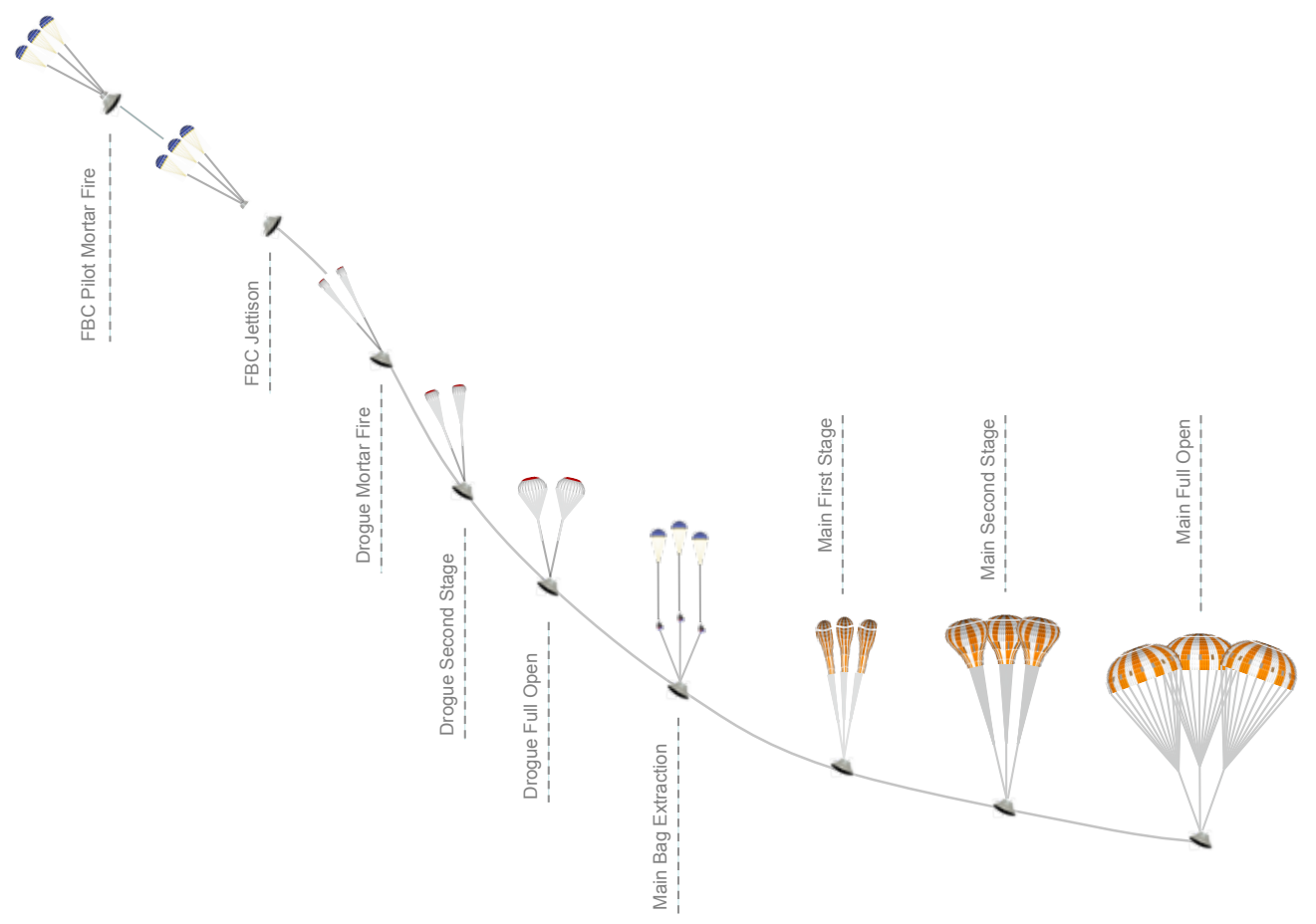

Figure 1. The CPAS parachute system includes FBC Pilots, Drogues, Main Pilots, and Mains.

The time between drogue release and the beginning of the inflation of the first stage of the mains is about $3.1 \mathrm{~s}$. During this time, there is no restoring force from a parachute, and the MPCV is in free flight, 
as illustrated in Figure 2. Not only is the MPCV generally dynamically unstable at low Mach numbers, but there is a large spike in the dynamic stability derivative, $C_{m_{q}}$, near the hang angle under the drogues and the free flight trim angle of attack, exactly where the vehicle will be flying during the deployment of the mains, as seen in Figure 3. Using an altitude trigger alone for drogue release can result in unfavorable attitudes and angular velocities at drogue release and cause the MPCV to tumble apex forward. This can complicate the extraction of the main parachute bags, which can become entrapped in the forward bay of the MPCV if the attitude is too far from heat shield forward. It also increases the risk of an extreme attitude when the main parachutes begin inflation, potentially dragging the main risers against structural components of the MPCV. This can damage a variety of structural elements in the forward bay, including the pitch RCS thrusters, which could then leak propellant and cause a hazard to the inflated chutes and to the recovery team. It can also sever the riser, as was seen in the Cluster Development Test 2 (CDT-2) CPAS drop. ${ }^{1}$

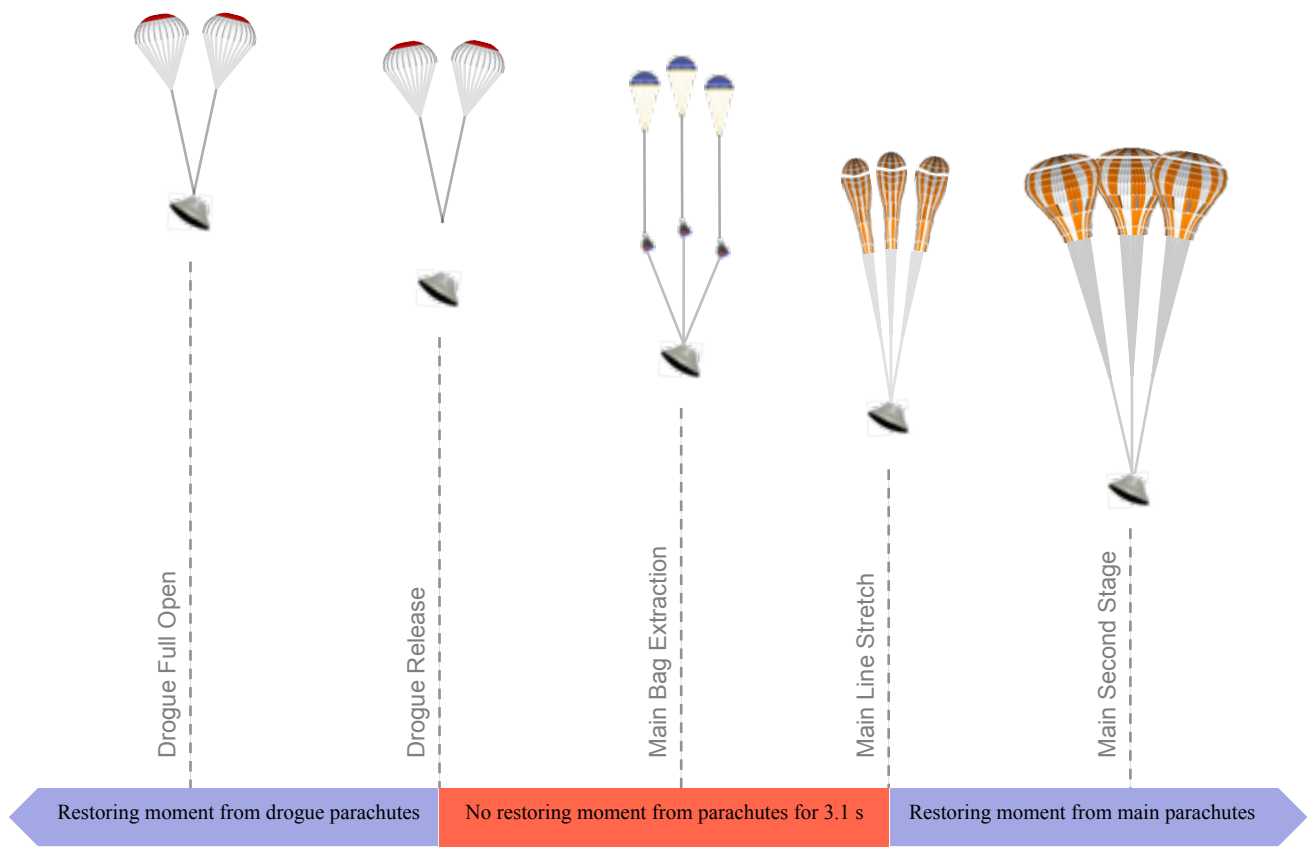

Figure 2. There are approximately $3.1 \mathrm{~s}$ of capsule free flight during the main parachute deploy sequence.

The concern of tumbling apex forward first motivated the development of a smart drogue release algorithm during the design of the Pad Abort (PA) 1 test. The baseline algorithm is simple in design, and only requires angular velocity knowledge. Pre-test Monte Carlo analysis showed that the algorithm provided a dramatic improvement in the number of cases flipping apex forward. ${ }^{2}$ Without smart drogue release, using just a time based release trigger, $51 \%$ of the cases flipped apex forward. With smart drogue release, only $0.1 \%$ tumbled. Post test analysis confirmed that the algorithm worked as expected. ${ }^{3}$

Smart drogue release was later used for CPAS drop tests and also incorporated into the mainline MPCV design. It will be flown on the upcoming EFT-1 test. It continues to perform well, and its simplicity remains an attractive quality. However, in cases where the attitude oscillations are large, the baseline algorithm can fail to prevent the MPCV from tumbling.

The main goal of this paper is to develop a new smart drogue release algorithm. The proposed algorithm is a numeric predictor, and integrates simplified equations of motion to find where a rotational energy metric is minimized. The simplified dynamics are developed in Section II and are validated through comparison to CPAS drop test data. The dynamics are then further simplified to develop decoupled second order differential equations, which provide closed form solutions for the angle of attack and sideslip angle motions. While not accurate enough for use in the predictor, the equations are useful for estimating the natural frequencies and constructing phase plane diagrams to illustrate the various smart drogue release algorithm concepts.

Three smart drogue release algorithms are then discussed in Section III. First, the baseline algorithm is discussed, and its advantages and disadvantages are enumerated. Then, a simple modification which improves the baseline algorithm in some cases is discussed. Finally, the numeric predictor algorithm is developed. Section IV compares the performance of the three algorithms, and also investigates the sensitivity of the 


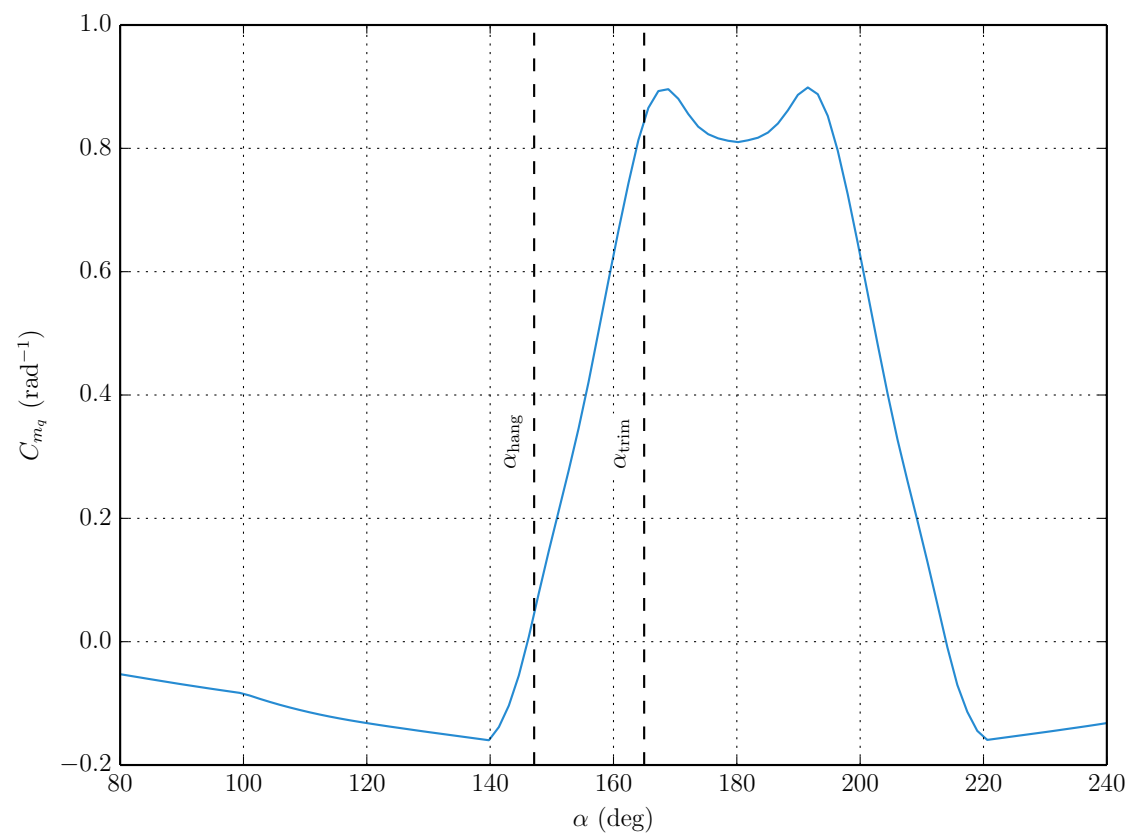

Figure 3. There is an unstable $C_{m_{q}}$ spike near the hang angle under the drogues and the free flight trim angle.

algorithms to aerodynamic dispersions, errors in the navigated state, and execution rate.

\section{Models}

\section{II.A. Aerodynamics}

For the drogue release problem the primary concern is the rotational dynamics of the MPCV at a Mach number between 0.2 and 0.3 over a short period of time of no more than $10 \mathrm{~s}$. Over such a short time frame, the motion will only complete a small number of oscillation cycles, and so the dynamic aerodynamic moments will be a second order effect and can be neglected. And at such a low Mach number, the variation of the static aerodynamic moments with Mach number can also be neglected. This results in a simple model consisting of the static pitching moment as a function of angle of attack and the static yawing moment as a function of sideslip angle. The curves in Figure 4 were generated using v0.71.1 of the MPCV aerodynamic database and representative MPCV EM-2 mass properties.

The trim angle of attack is $165 \mathrm{deg}$. The trim sideslip angle is very near zero, at $0.295 \mathrm{deg}$. Both moment coefficients are nearly linear in the region around the trim angles. The slopes of the linear regions are $C_{m_{\alpha}}=-0.111 \mathrm{rad}^{-1}$ and $C_{n_{\beta}}=-0.107 \mathrm{rad}^{-1}$.

\section{II.B. Parachutes}

A simple but accurate model of a low mass ratio parachute system such as the CPAS drogue parachutes was developed during the Apollo program. The model has recently been studied further by the Orion program. Photogrammetric analysis of the PA- 1 test $^{4}$ and testing at the NASA Langley Research Center Vertical Spin Tunnel ${ }^{5}$ both validate the model.

The magnitude of the parachute force is computed using the drag area of the parachute, but the vector along which the force is applied opposes the velocity of the attach point itself, such that the force vector accounts for the motion of the attach point due to the rotational motion of the MPCV. It is believed that this effect is caused by the riser rotating as the attach point moves, causing a hysteresis in the moment arm. 

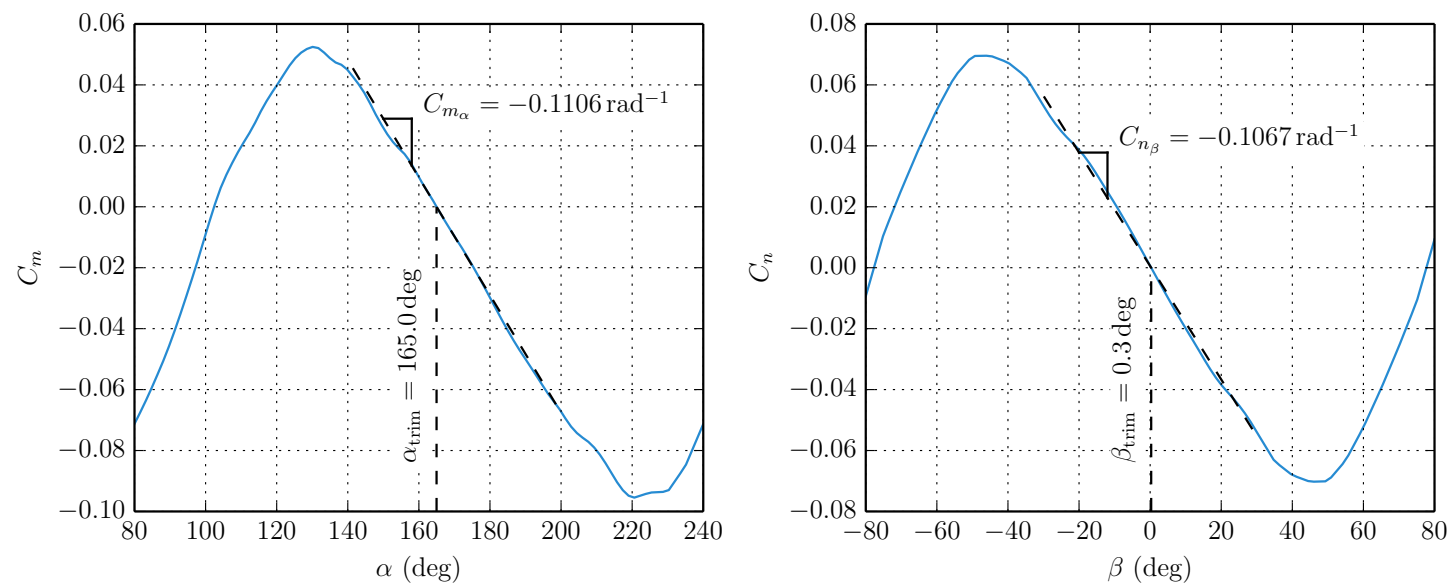

Figure 4. Static pitching moment as a function of angle of attack and static yawing moment as a function of sideslip angle.

This is illustrated in Figure 5. The parachute force is:

$$
\underline{F}_{\text {chute }}=-q\left(C_{D} S\right)_{\text {chute }} \frac{\underline{v}_{\infty}+\underline{\omega} \times \underline{R}}{\left\|\underline{v}_{\infty}+\underline{\omega} \times \underline{R}\right\|}
$$

Where $\underline{R}$ is the vector from the center of gravity to the attach point.
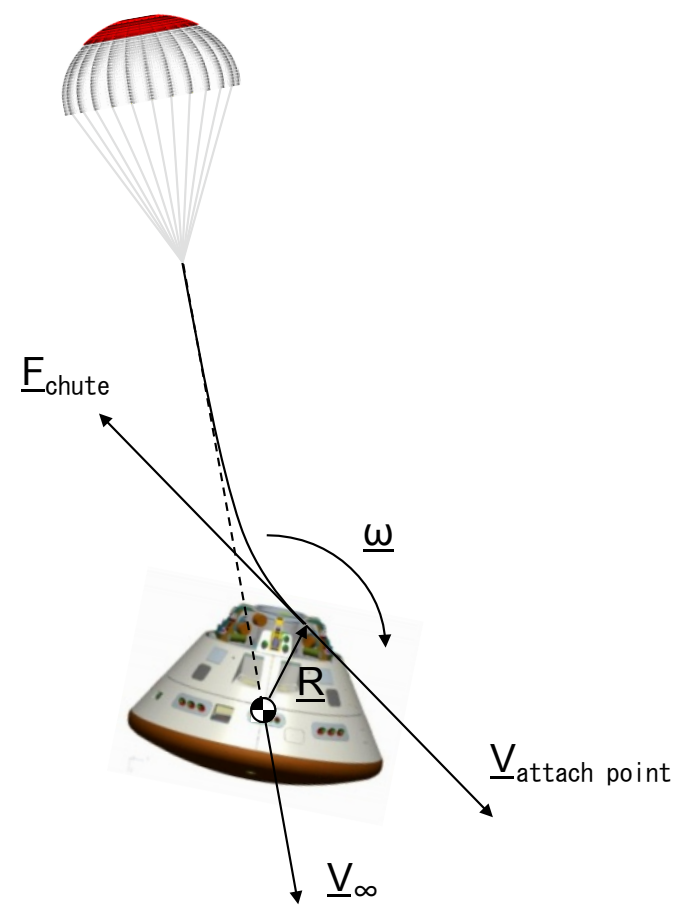

Figure 5. The parachute force vector includes a damping component from the vehicle rotation.

\section{II.C. Dynamics}

A set of simplified rotational equations of motion were developed. The state vector consists of $\alpha, \beta, \omega_{x}$, $\omega_{y}$, and $\omega_{z}$. Note that since the translational state is not included, the dynamic pressure is assumed to be 
constant. Since the equations will be applied to the motion of the MPCV at the end of the ride under the drogues, the system will be at steady state, and this assumption is valid.

For the kinematic equations, the rotation of the planet and the derivatives of longitude, latitude, azimuth, and flight path angle are neglected. For flight after reaching steady state under the drogues, the flight path angle will be nearly vertical, and so these assumptions are valid. The angular velocity is then only a function of the change of the rotation from the freestream velocity frame to the body frame. This rotation can be represented as an Euler sequence consisting of a positive roll rotation by the bank angle, a negative yaw rotation by the sideslip angle, and a positive pitch rotation by the angle of attack:

$$
\mathbf{R}=\mathbf{R}_{\phi} \mathbf{R}_{-\beta} \mathbf{R}_{\alpha}
$$

The angular velocity in the body frame, expressed in tensor form, is thus:

$$
\begin{aligned}
\widetilde{\omega} & =\mathbf{R}^{\mathrm{T}} \dot{\mathbf{R}} \\
& =\left(\mathbf{R}_{\phi} \mathbf{R}_{-\beta} \mathbf{R}_{\alpha}\right)^{\mathrm{T}}\left(\dot{\mathbf{R}}_{\phi} \mathbf{R}_{-\beta} \mathbf{R}_{\alpha}+\mathbf{R}_{\phi} \dot{\mathbf{R}}_{-\beta} \mathbf{R}_{\alpha}+\mathbf{R}_{\phi} \mathbf{R}_{-\beta} \dot{\mathbf{R}}_{\alpha}\right) \\
& =\mathbf{R}_{\alpha}^{\mathrm{T}} \mathbf{R}_{-\beta}^{\mathrm{T}} \mathbf{R}_{\phi}^{\mathrm{T}} \dot{\mathbf{R}}_{\phi} \mathbf{R}_{-\beta} \mathbf{R}_{\alpha}+\mathbf{R}_{\alpha}^{\mathrm{T}} \mathbf{R}_{-\beta}^{\mathrm{T}} \dot{\mathbf{R}}_{-\beta} \mathbf{R}_{\alpha}+\mathbf{R}_{\alpha}^{\mathrm{T}} \dot{\mathbf{R}}_{\alpha}
\end{aligned}
$$

Where the $\mathbf{R}^{\mathrm{T}} \dot{\mathbf{R}}$ terms are simply the tensor form of the derivatives of the individual Euler angles in their respective intermediate frames. Converting from a tensor equation to a vector equation, the angular velocity in the body frame is:

$$
\begin{aligned}
\underline{\omega} & =\mathbf{R}_{\alpha}^{\mathrm{T}} \mathbf{R}_{-\beta}^{\mathrm{T}}\left[\begin{array}{l}
\dot{\phi} \\
0 \\
0
\end{array}\right]+\mathbf{R}_{\alpha}^{\mathrm{T}}\left[\begin{array}{c}
0 \\
0 \\
-\dot{\beta}
\end{array}\right]+\left[\begin{array}{c}
0 \\
\dot{\alpha} \\
0
\end{array}\right] \\
& =\left[\begin{array}{ccc}
\cos \alpha & 0 & -\sin \alpha \\
0 & 1 & 0 \\
\sin \alpha & 0 & \cos \alpha
\end{array}\right]\left[\begin{array}{ccc}
\cos -\beta & \sin -\beta & 0 \\
-\sin -\beta & \cos -\beta & 0 \\
0 & 0 & 1
\end{array}\right]\left[\begin{array}{c}
\dot{\phi} \\
0 \\
0
\end{array}\right]+\left[\begin{array}{ccc}
\cos \alpha & 0 & -\sin \alpha \\
0 & 1 & 0 \\
\sin \alpha & 0 & \cos \alpha
\end{array}\right]\left[\begin{array}{c}
0 \\
0 \\
-\dot{\beta}
\end{array}\right]+\left[\begin{array}{c}
0 \\
\dot{\alpha} \\
0
\end{array}\right] \\
& =\left[\begin{array}{c}
\dot{\phi} \cos \alpha \cos \beta+\dot{\beta} \sin \alpha \\
\dot{\phi} \sin \beta+\dot{\alpha} \\
\dot{\phi} \sin \alpha \cos \beta-\dot{\beta} \cos \alpha
\end{array}\right]
\end{aligned}
$$

Which can be solved for $\dot{\alpha}$ and $\dot{\beta}$ as functions of the angular velocity components:

$$
\begin{aligned}
& \dot{\alpha}=\omega_{y}-\left(\omega_{x} \cos \alpha+\omega_{z} \sin \alpha\right) \tan \beta \\
& \dot{\beta}=\omega_{x} \sin \alpha-\omega_{z} \cos \alpha
\end{aligned}
$$

The kinetics are simply Euler's equations. Because the MPCV is roughly axially symmetric, the products of inertia are neglected. The angular accelerations are thus:

$$
\begin{aligned}
& \dot{\omega}_{x}=\frac{M_{x}+\omega_{y} \omega_{z}\left(I_{y y}-I_{z z}\right)}{I_{x x}} \\
& \dot{\omega}_{y}=\frac{M_{y}+\omega_{x} \omega_{z}\left(I_{z z}-I_{x x}\right)}{I_{y y}} \\
& \dot{\omega}_{z}=\frac{M_{z}+\omega_{x} \omega_{y}\left(I_{x x}-I_{y y}\right)}{I_{z z}}
\end{aligned}
$$

The moments are the sum of the static aerodynamic moments and the moment applied by the parachutes:

$$
\underline{M}=q S c\left[\begin{array}{c}
0 \\
C_{m} \\
C_{n}
\end{array}\right]+\underline{R} \times \underline{F}_{\text {chute }}
$$

Where $C_{m}$ and $C_{n}$ are functions of $\alpha$ and $\beta$, respectively. 
This simplified model of the dynamics was compared against a simulation of an EM-2 entry and flight data from two CPAS drop tests, CDT 3-9 and CDT 3-11. All comparisons showed a favorable match. Figure 6 shows a comparison of the simplified dynamics to the CDT 3-11 Best Estimated Trajectory (BET). The time data used in the plots are referenced to when the pallet to which the test article was attached exited the aircraft.
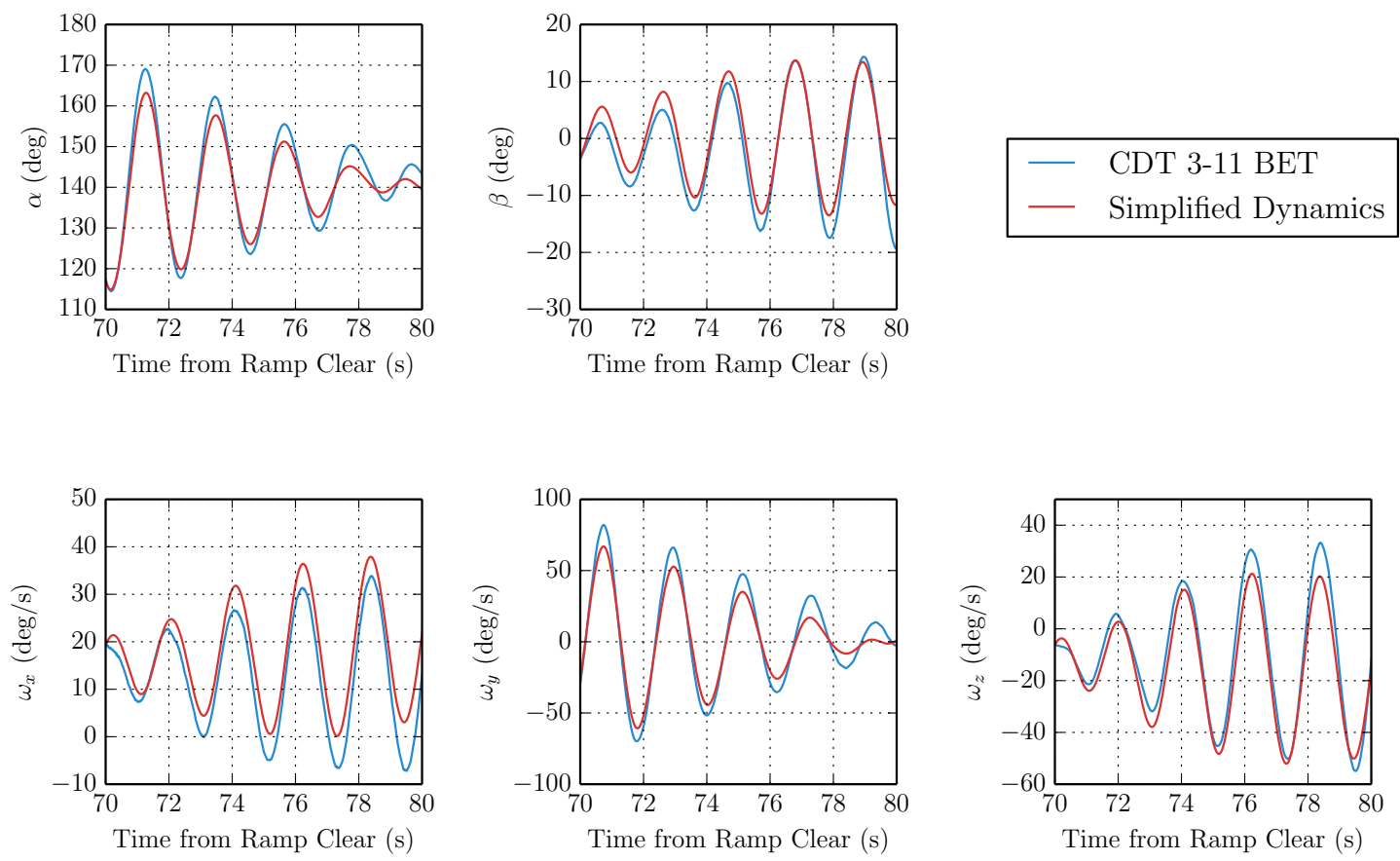

Figure 6. Comparison of the CDT 3-11 BET and the simplified dynamics.

\section{II.D. Closed Form Solutions to the Dynamics}

With a few additional simplifications, closed form solutions to the dynamics can be developed. As shown above, the static aerodynamic moment coefficients vary nearly linearly in the vicinity of the trim angles. By assuming $C_{m_{\alpha}}$ and $C_{n_{\beta}}$ are constant, the aerodynamic moments become:

$$
\underline{M}_{\text {aero }}=q S c\left[\begin{array}{c}
0 \\
C_{m_{\alpha}}\left(\alpha-\alpha_{\text {trim }}\right) \\
C_{n_{\beta}}\left(\beta-\beta_{\text {trim }}\right)
\end{array}\right]
$$

The parachute moment can also be simplified. First, note that the freestream velocity in the body frame can be written as:

$$
\underline{v}_{\infty}=v_{\infty}\left[\begin{array}{c}
\cos \beta \cos \alpha \\
\sin \beta \\
\cos \beta \sin \alpha
\end{array}\right]
$$

Neglecting the damping term due to the motion of the attach point, the parachute force simply opposes the freestream velocity, and can be written as:

$$
\underline{D}=-q\left(C_{D} S\right)_{\text {chute }}\left[\begin{array}{c}
\cos \beta \cos \alpha \\
\sin \beta \\
\cos \beta \sin \alpha
\end{array}\right]
$$


When the vehicle is hanging still under a parachute, it will be at a certain angle of attack and sideslip angle. In this configuration, the vector from the center of gravity to the parachute attach point will also oppose the velocity vector:

$$
\underline{R}=-R\left[\begin{array}{c}
\cos \beta_{\text {hang }} \cos \alpha_{\text {hang }} \\
\sin \beta_{\text {hang }} \\
\cos \beta_{\text {hang }} \sin \alpha_{\text {hang }}
\end{array}\right]
$$

Where $R$ is the magnitude of the vector from the center of gravity to the attach point. For the representative EM-2 MPCV configuration, $\alpha_{\text {hang }}=147 \mathrm{deg}$ and $\beta_{\text {hang }}=1.26 \times 10^{-3} \mathrm{deg}$. The sideslip angle is assumed to be zero to yield:

$$
\underline{R}=-R\left[\begin{array}{c}
\cos \alpha_{\text {hang }} \\
0 \\
\sin \alpha_{\text {hang }}
\end{array}\right]
$$

The parachute moments are thus:

$$
\underline{M}_{\text {chute }}=\underline{R} \times \underline{D}
$$

The rolling moment can be written as:

$$
\begin{aligned}
M_{\text {chute }, x} & =-\left(-R \sin \alpha_{\text {hang }}\right)\left(-q\left(C_{D} S\right)_{\text {chute }} \sin \beta\right) \\
& =-q\left(C_{D} S\right)_{\text {chute }} R \sin \alpha_{\text {hang }} \sin \beta
\end{aligned}
$$

And assuming that $\beta$ is a small angle yields:

$$
M_{\text {chute }, x}=-q\left(C_{D} S\right)_{\text {chute }} R \sin \left(\alpha_{\text {hang }}\right) \beta
$$

The pitching moment can be written as:

$$
\begin{aligned}
M_{\text {chute }, y} & =\left(-R \sin \alpha_{\text {hang }}\right)\left(-q\left(C_{D} S\right)_{\text {chute }} \cos \beta \cos \alpha\right)-\left(-R \cos \alpha_{\text {hang }}\right)\left(-q\left(C_{D} S\right)_{\text {chute }} \cos \beta \sin \alpha\right) \\
& =q\left(C_{D} S\right)_{\text {chute }} R \cos \beta\left(\sin \alpha_{\text {hang }} \cos \alpha-\cos \alpha_{\text {hang }} \sin \alpha\right)
\end{aligned}
$$

Applying the following identity:

$$
\sin (u-v)=\sin u \cos v-\cos u \sin v
$$

The pitching moment can be reduced to:

$$
M_{\text {chute }, y}=-q\left(C_{D} S\right)_{\text {chute }} R \cos \beta \sin \left(\alpha-\alpha_{\text {hang }}\right)
$$

Decoupling by assuming $\beta$ is zero and assuming that $\alpha-\alpha_{\text {hang }}$ is a small angle yields:

$$
M_{\text {chute }, y}=-q\left(C_{D} S\right)_{\text {chute }} R\left(\alpha-\alpha_{\text {hang }}\right)
$$

Finally, the yawing moment is written as:

$$
\begin{aligned}
M_{\text {chute }, z} & =\left(-R \cos \alpha_{\text {hang }}\right)\left(-q\left(C_{D} S\right)_{\text {chute }} \sin \beta\right) \\
& =q\left(C_{D} S\right)_{\text {chute }} R \cos \alpha_{\text {hang }} \sin \beta
\end{aligned}
$$

And again assuming that $\beta$ is a small angle:

$$
M_{\text {chute }, z}=q\left(C_{D} S\right)_{\text {chute }} R \cos \left(\alpha_{\text {hang }}\right) \beta
$$

The kinetics and kinematics can also be simplified. First, note that $I_{y y}$ and $I_{z z}$ are similar for a vehicle symmetric about the $x$ axis. Also assume that $\omega_{x}$ is small, which is not necessarily true for the MPCV but is convenient here. These two assumptions allow the coupling terms to be neglected such that:

$$
\begin{aligned}
& \dot{\omega}_{x}=\frac{M_{x}}{I_{x x}} \\
& \dot{\omega}_{y}=\frac{M_{y}}{I_{y y}} \\
& \dot{\omega}_{z}=\frac{M_{z}}{I_{z z}}
\end{aligned}
$$

$$
8 \text { of } 21
$$


Beginning with the expression for $\dot{\alpha}$ in Equation 5, decoupling it by assuming $\beta$ is zero and then taking a derivative yields:

$$
\begin{aligned}
\ddot{\alpha} & =\dot{\omega}_{y} \\
& =\frac{M_{y}}{I_{y y}}
\end{aligned}
$$

Similarly, starting with the expression for $\dot{\beta}$ in Equation 5, decoupling the equation by assuming $\alpha$ is $\alpha_{\text {hang }}$ and taking a derivative gives:

$$
\begin{aligned}
\ddot{\beta} & =\dot{\omega}_{x} \sin \alpha_{\text {hang }}-\dot{\omega}_{z} \cos \alpha_{\text {hang }} \\
& =\frac{M_{x}}{I_{x x}} \sin \alpha_{\text {hang }}-\frac{M_{z}}{I_{z z}} \cos \alpha_{\text {hang }}
\end{aligned}
$$

After substituting the aerodynamic and parachute moments into these equations, they can be rearranged in the form of a harmonic oscillator:

$$
\begin{aligned}
\ddot{\alpha}+A \alpha & =B \\
A & =\frac{q\left(C_{D} S\right)_{\text {chute }} R}{I_{y y}}-\frac{q S c C_{m_{\alpha}}}{I_{y y}} \\
B & =\frac{q\left(C_{D} S\right)_{\text {chute }} R}{I_{y y}} \alpha_{\text {hang }}-\frac{q S c C_{m_{\alpha}}}{I_{y y}} \alpha_{\text {trim }} \\
\ddot{\beta}+C \beta & =0 \\
C & =\frac{q\left(C_{D} S\right)_{\text {chute }} R \sin ^{2} \alpha_{\text {hang }}}{I_{x x}}+\frac{q\left(C_{D} S\right)_{\text {chute }} R \cos ^{2} \alpha_{\text {hang }}}{I_{z z}}+\frac{q S c C_{n_{\beta}} \cos \alpha_{\text {hang }}}{I_{z z}}
\end{aligned}
$$

From these equations the natural frequencies and periods of the angle of attack and sideslip angle motion can be calculated as:

$$
\begin{aligned}
\omega_{n, \alpha} & =\sqrt{A} & \omega_{n, \beta} & =\sqrt{C} \\
T_{\alpha} & =\frac{2 \pi}{\omega_{n, \alpha}} & T_{\beta} & =\frac{2 \pi}{\omega_{n, \beta}}
\end{aligned}
$$

This can be compared to the same CDT 3-11 BET data that was used above for validating the integration of the simplified dynamics. With the parameters for that drop test, the frequencies and periods are estimated to be:

$$
\begin{aligned}
\omega_{n, \alpha} & =0.458 \mathrm{~Hz} & \omega_{n, \beta} & =0.454 \mathrm{~Hz} \\
T_{\alpha} & =2.18 \mathrm{~s} & T_{\beta} & =2.20 \mathrm{~s}
\end{aligned}
$$

A Fast Fourier Transform was applied to the BET data to identify the dominant frequencies. The results are shown in terms of the period in Figure 7 . The dominant period is just above $2 \mathrm{~s}$ for both angle of attack and sideslip angle, which is consistent with the estimated values. Since any smart drogue release algorithm works by timing the release of the drogues during the natural oscillation of the rotational state, the period provides a way of selecting a drogue release window length. The analyst can select the number of oscillation cycles over which the algorithm should operate. Clearly the minimum window length is one period. The estimated frequencies thus provide a way of adjusting the window length if the vehicle parameters change, such as for abort cases where the drogue parachutes have not yet fully disreefed.

The harmonic oscillator equations have closed form solutions for angle of attack and sideslip angle:

$$
\begin{aligned}
& \alpha=c_{1} \sin \sqrt{A} t+c_{2} \cos \sqrt{A} t+\frac{B}{A} \\
& \beta=c_{3} \sin \sqrt{C} t+c_{4} \cos \sqrt{C} t
\end{aligned}
$$

Given $\alpha(0)=\alpha_{0}, \dot{\alpha}(0)=\dot{\alpha}_{0}, \beta(0)=\beta_{0}$, and $\dot{\beta}(0)=\dot{\beta}_{0}$, the equations are:

$$
\begin{aligned}
& \alpha=\frac{\dot{\alpha}_{0}}{\sqrt{A}} \sin \sqrt{A} t+\left(\alpha_{0}-\frac{B}{A}\right) \cos \sqrt{A} t+\frac{B}{A} \\
& \beta=\frac{\dot{\beta}_{0}}{\sqrt{C}} \sin \sqrt{C} t+\beta_{0} \cos \sqrt{C} t
\end{aligned}
$$



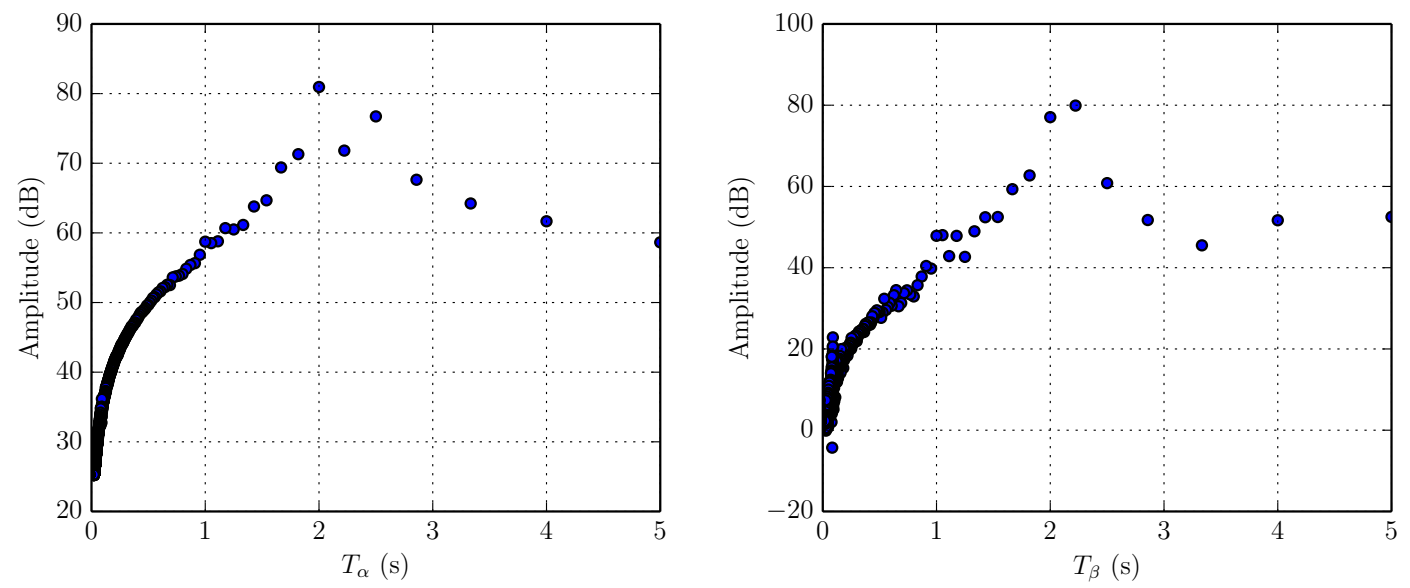

Figure 7. Comparison of the periods from CDT 3-11 BET and the closed form dynamics.

Comparing the values computed by these equations for the drogue ride of CDT 3-11 to the BET of that drop test, shown in Figure 8, it is clear that the equations are not accurate enough to properly reconstruct the motion. This is mainly due to neglecting the damping and the interaction between the axes. However, the equations can be used to generate phase plane diagrams of motion constrained to a single axis for both flight under parachutes and in free flight, simply by setting the $\left(C_{D} S\right)_{\text {chute }}$ term to zero. These diagrams will be used extensively as the various smart drogue release algorithms are discussed in the following section.
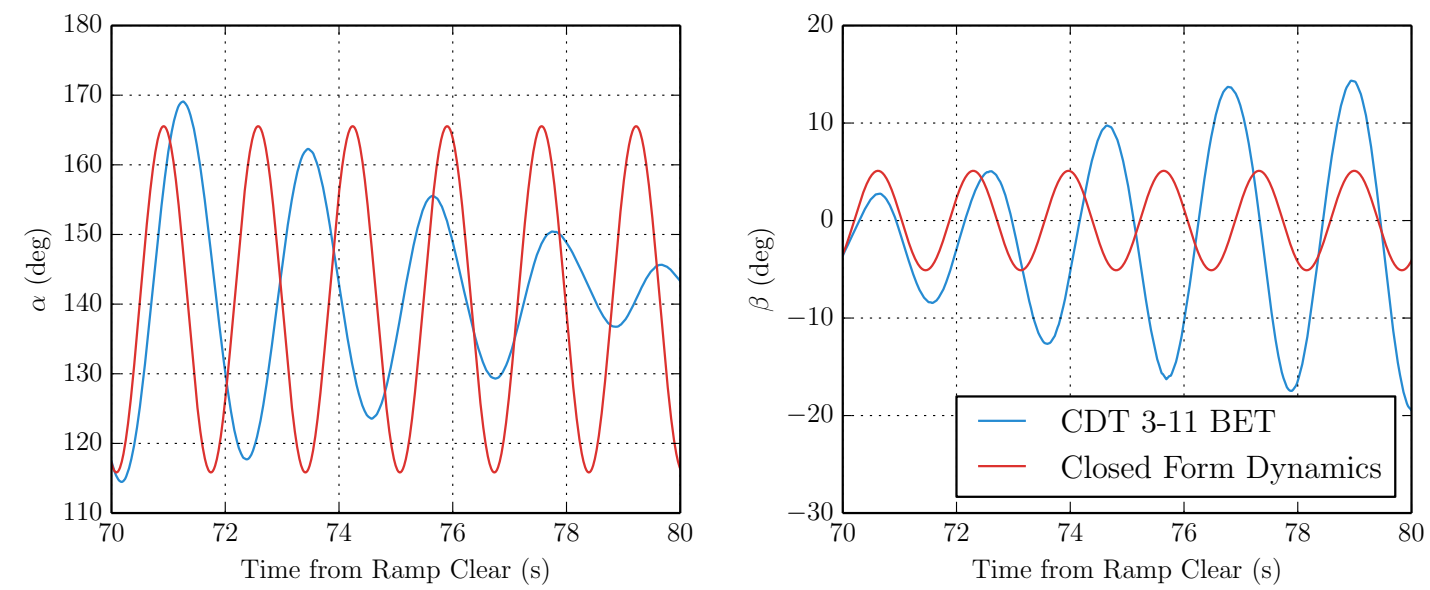

Figure 8. Comparison of the CDT 3-11 BET and the closed form dynamics.

\section{Algorithms}

\section{III.A. Baseline: Minimum RSS Rate}

The baseline algorithm, originally developed for PA-1 and now being used for EFT-1, is based on trying to minimize the RSS of the pitch and yaw rates at drogue release. Consider the case when the motion is restricted to the pitch plane. The phase plane in Figure 9 contrasts two drogue release scenarios. Clearly the case that releases when the RSS rate is minimized (in this case, when pitch rate is zero) results in a lower amplitude oscillation in free flight.

There is a latency of approximately $70 \mathrm{~ms}$ between the flight computer sending the drogue release command and the cutters physically separating the drogue parachutes. While the period of the oscillation of 

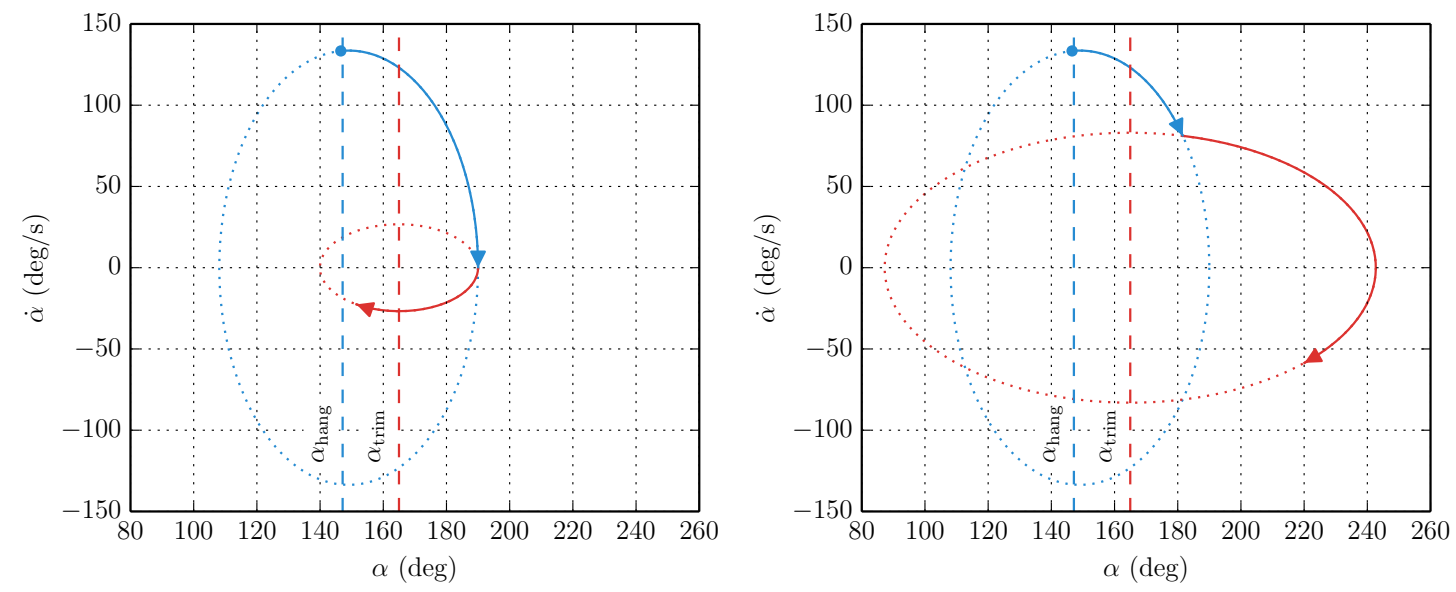

Figure 9. Drogue release at two different states.

angle of attack was calculated above to be $2.18 \mathrm{~s}$, the period of the oscillation of the RSS rate is half that, since it is effectively an absolute value. So the latency is nearly one tenth of the full period. If the algorithm were to release at the actual minimum, by the time the release physically occurred, the RSS rate would have increased substantially and the effectiveness of the algorithm would be reduced.

To account for this, the algorithm does not try to detect the actual local minimum of the oscillation. Instead, it records the most recent peak and trough of the oscillation. The current RSS rate is then compared to the peak and trough values to estimate where it falls in the oscillation, with $100 \%$ meaning the current RSS rate is equal to the previous peak and 0\% meaning the current RSS is equal to the previous trough. When the percentage is decreasing and reduces below $35 \%$, the algorithm anticipates the approaching local minimum and the release is commanded. This is illustrated in Figure 10. The trigger value of 35\% was tuned through a Monte Carlo analysis in order to optimize the performance of the algorithm.

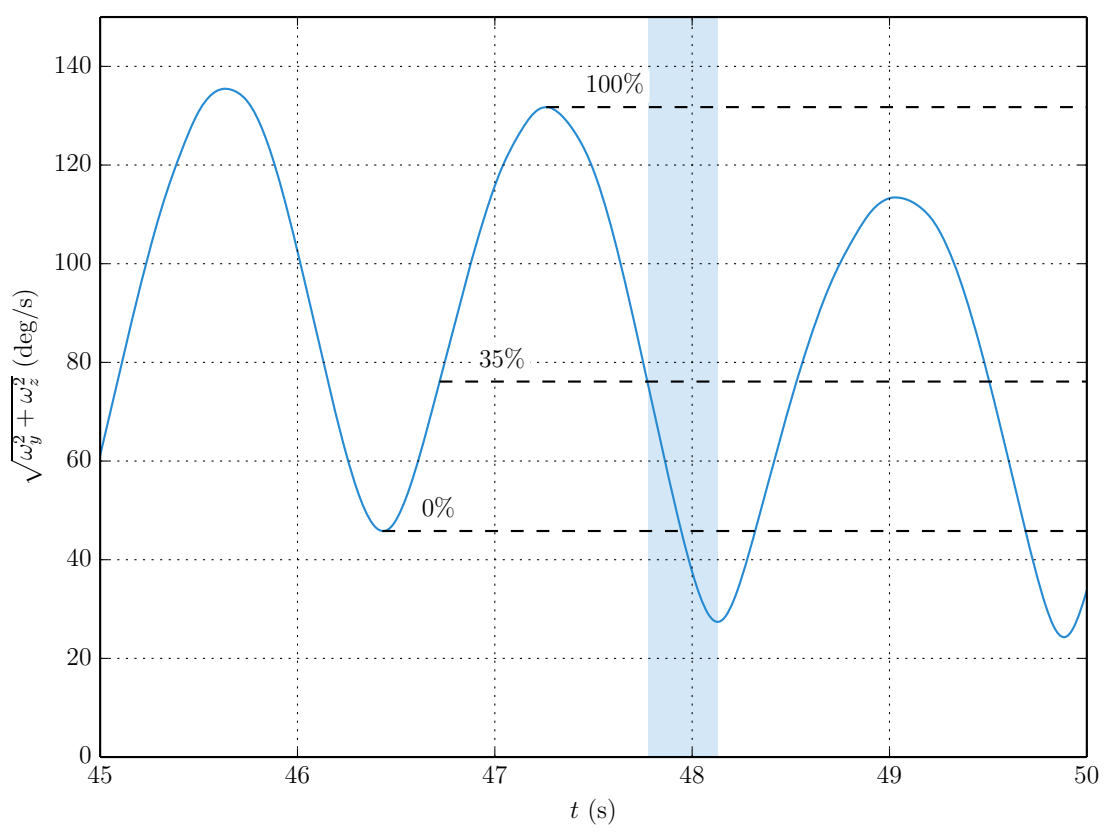

Figure 10. The RSS rate condition is satisfied when the RSS rate percentage is decreasing and is below $35 \%$. 
This algorithm is simple and very often effective. The logic requires only a few lines of code. The data needs are few, with the algorithm requiring only the pitch and yaw rates from the navigation system. However, the performance of the algorithm begins to break down when the angle of attack oscillation under the drogue parachutes becomes too large. This can occur if the dynamic aerodynamics of the payload vehicle are unfavorable or if one of the drogue parachutes has failed, such that the parachute cluster is providing less damping. Consider again the case where the motion is restricted to the pitch plane, now with a drogue failed. Because the free flight trim angle is offset from the hang angle under the drogues, the two points where pitch rate is zero give different performance. The one closer to the free flight trim angle results in a smaller amplitude motion after drogue release. This is shown in Figure 11.

In addition, the oscillations under the drogues can be so large that the point on the low side of angle of attack where pitch rate is zero can be outside the region of static stability. Recall the pitching moment curve in Figure 4. The hang angle under the drogues, at $147 \mathrm{deg}$, is much closer to the static stability limit on the low side of angle of attack, at about $100 \mathrm{deg}$, than the limit on the high side, at about $260 \mathrm{deg}$.
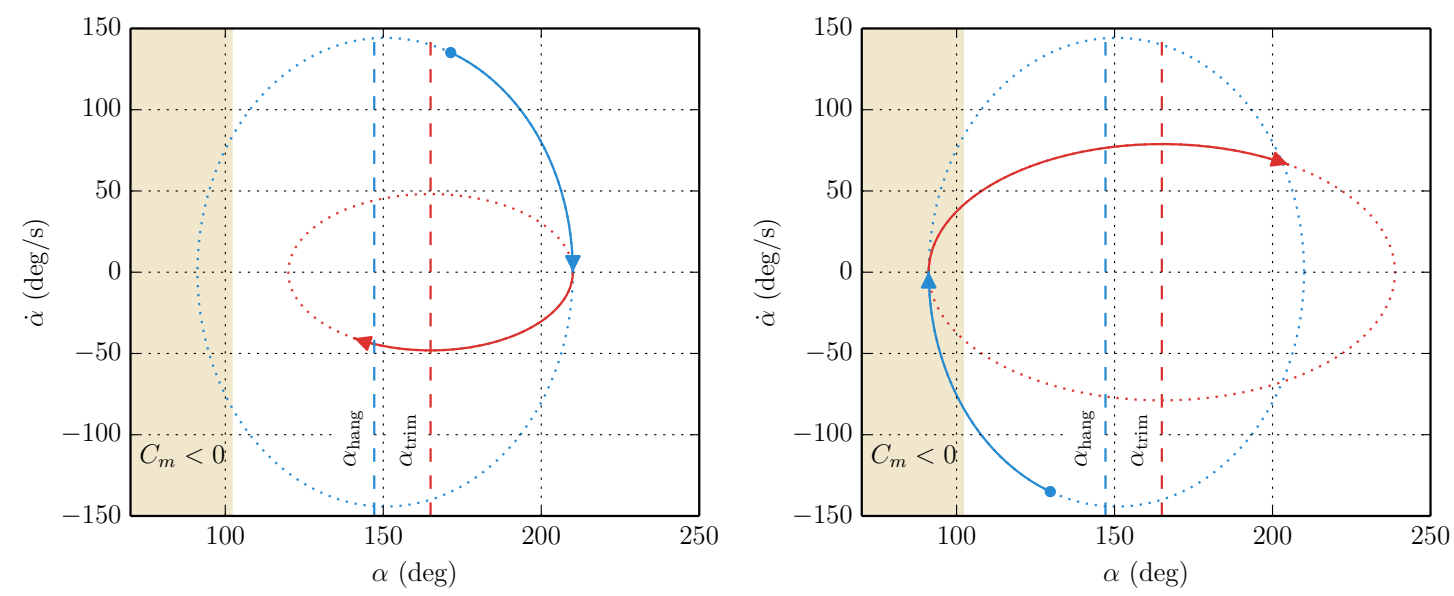

Figure 11. Drogue release at the two zero rate points.

\section{III.B. Modified Baseline: Minimum RSS Rate with Pitch Rate Condition}

The CPAS CDT 3-7 drop test utilized a single drogue parachute to simulate a drogue parachute failure. Making matters worse, this test used the Parachute Test Vehicle (PTV), which has a similar wake to the MPCV but is even more dynamically unstable. While doing the pre-flight analysis for this test, it became clear that the large angle of attack oscillations were stressing the baseline algorithm, with $36 \%$ of the Monte Carlo cases flipping apex forward. ${ }^{6}$

A modification to the baseline algorithm was developed to account for the difference between a minimum RSS rate at a low angle of attack and one at a high angle of attack, nearer to the free flight trim condition. The modification is based on the fact that when the angle of attack is greater than the hang angle, the pitch rate is decreasing. This is clear from the phase plane in Figure 11.

The modified algorithm tracks the RSS of pitch and yaw rate the same way as the baseline algorithm. It also similarly tracks the pitch rate, recording the most recent minimum and maximum pitch rate. The current pitch rate is compared to these values to again compute a percentage, going from $-100 \%$ at the minimum value to $100 \%$ at the maximum value. When the pitch rate is decreasing and is between $50 \%$ and $-50 \%$, the pitch rate condition is satisfied. This is shown in Figure 12. When both the RSS rate condition and the pitch rate condition are simultaneously satisfied, the drogue release is commanded.

An advantage of this algorithm is that it is a fairly straightforward way to augment the baseline algorithm and incorporate the additional information about how the hang angle and free flight trim angles are offset from each other. Since it uses the pitch rate, it requires no new information from the navigation system. In combination with several other mitigations, the application of this modified algorithm to the CDT 3-7 preflight Monte Carlo reduced the failure rate to less than $1 \%$ and enabled the test to be approved. ${ }^{6}$ Post-flight 


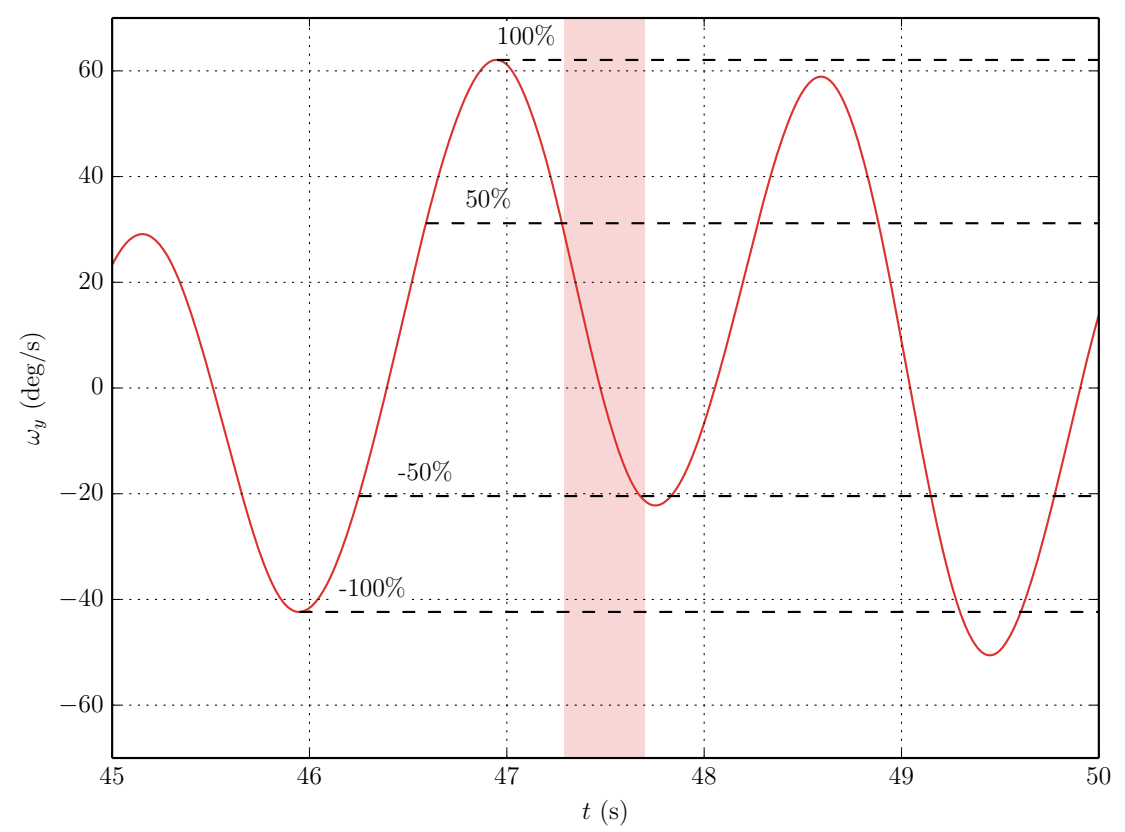

Figure 12. The pitch rate condition is satisfied when the pitch rate percentage is increasing and between $-50 \%$ and $50 \%$.

reconstruction showed that the algorithm performed well, and released using both the RSS rate and pitch rate conditions. This algorithm is still used by CPAS for its drop tests.

However, the two trigger approach of the algorithm is a major disadvantage. It is possible for the motion to be such that the two conditions are not satisfied at the same time during the drogue release window. While flying under the drogue parachutes, the vehicle tends to twist around the parachute riser. Because the vehicle is at a hang angle, this causes a coupled motion in the roll and yaw axes. While the derivative of the sideslip angle oscillates about $0 \mathrm{deg}$, the roll and yaw rates do not, as shown in Figure 13. The yaw rate can actually have a large steady state value that can then dominate the RSS rate metric, and cause it to not be in phase with the pitch motion as one would expect from a simple rocking motion under the parachutes. The only way to increase the likelihood of the triggers overlapping is to relax the conditions. But this can degrade the performance of cases that were already working well. To make a best effort in cases where the conditions are out of phase, the modified algorithm reverts to the baseline algorithm after a specified time.

\section{III.C. Numeric Predictor to Minimize Energy}

In order to better incorporate the concepts of releasing at a minimum RSS rate and close to the free flight trim condition, a new metric based on energy was developed. Since the pitch and yaw dynamics are the first order drivers of the likelihood of the vehicle flipping apex forward, the energy metric is a sum of the energies in the pitch and yaw axes only. The smart drogue release algorithm can then select a release condition based on minimizing this energy metric.

The potential energy can be computed as an integral of the static aerodynamic moments from the respective trim condition to the current condition. This computation is simplified by assuming the static aerodynamic curves are linear, such that $C_{m_{\alpha}}$ and $C_{n_{\beta}}$ are constant. For example, the potential energy in 


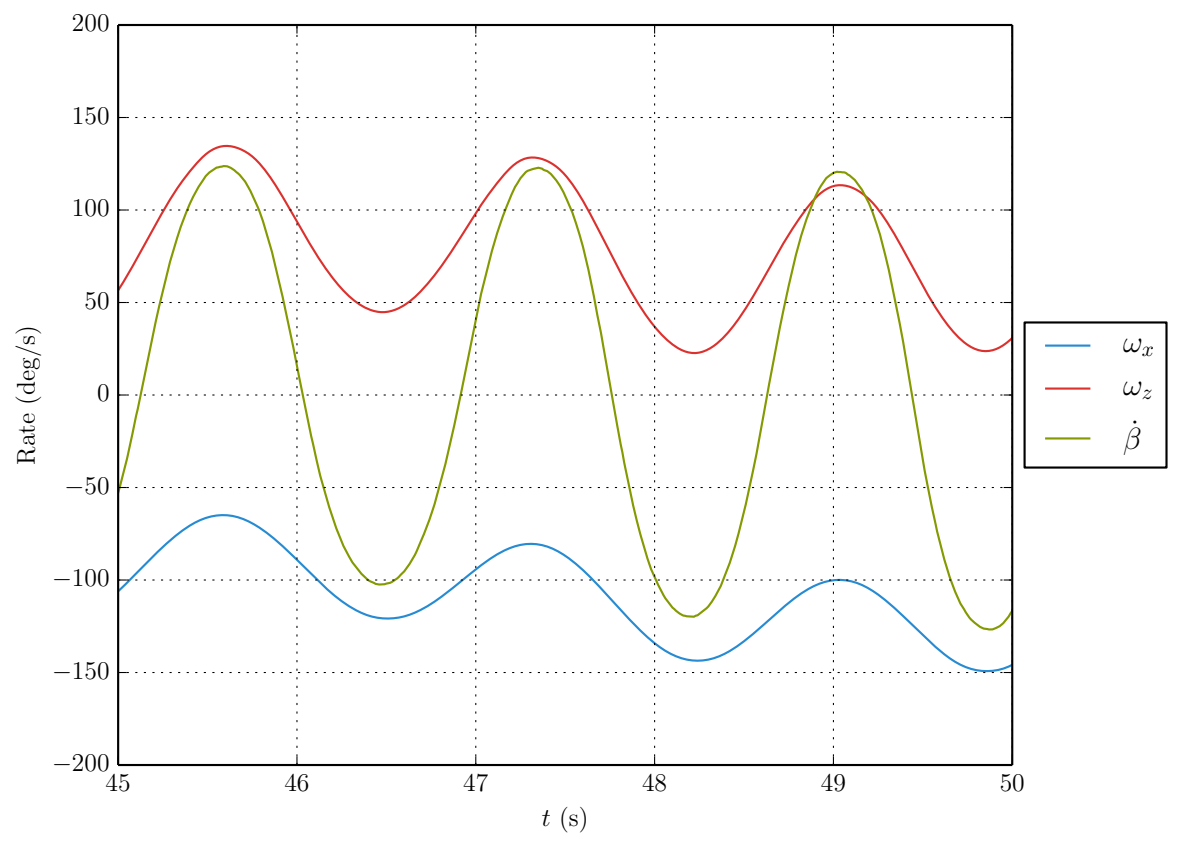

Figure 13. Comparison of roll rate, yaw rate, and sideslip angle rate.

the pitch axis is:

$$
\begin{aligned}
U_{y} & =-\int_{\alpha_{\text {trim }}}^{\alpha} M_{y} d \alpha \\
& =-\int_{\alpha_{\text {trim }}}^{\alpha} q S c C_{m} d \alpha \\
& =-\iint_{\alpha_{\text {trim }}}^{\alpha} q S c C_{m_{\alpha}} d \alpha^{2} \\
& \left.=-\frac{1}{2} q S c C_{m_{\alpha}} \alpha^{2}\right]_{\alpha_{\text {trim }}}^{\alpha} \\
& =-\frac{1}{2} q S c C_{m_{\alpha}}\left(\alpha-\alpha_{\text {trim }}\right)^{2}
\end{aligned}
$$

The total energy in the pitch and yaw axes is therefore:

$$
E=\frac{1}{2} q S c C_{m_{\alpha}}\left(\alpha-\alpha_{\text {trim }}\right)^{2}+\frac{1}{2} I_{y y} \omega_{y}^{2}+\frac{1}{2} q S c C_{n_{\beta}}\left(\beta-\beta_{\text {trim }}\right)^{2}+\frac{1}{2} I_{z z} \omega_{z}^{2}
$$

Again, this is the energy in the pitch and yaw axes, not in the alpha and beta directions. As was shown in Figure 13, the derivative of sideslip angle has components from both yaw and roll, because of the coupling caused by the drogue parachute riser. But once the drogue is released, that coupling is no longer enforced. The yaw rate is of primary concern, and it is clear that yaw rate is not necessarily minimized when the derivative of sideslip angle is zero. A fortunate side effect of this approach is that when the yaw rate is minimized, the energy has transferred to the roll channel and the roll rate is large. While it is more of a second order effect, a large roll rate is actually beneficial to preventing flipping apex forward.

This energy metric is not constant with time, and will oscillate for several reasons. First, the potential energy is referenced to the trim angles, but the attitude oscillation under the drogues is offset from the trim angle. Second, the energy of the roll axis is not included, but the roll and yaw axes are exchanging energy as the vehicle twists about the drogue riser.

Using this improved metric, the first attempts at creating a new smart drogue release algorithm applied optimal control theory with dynamic constraints specified using the closed form dynamics described in 
Section II.D. However, these early attempts faced two major issues. First of all, as was shown above, the closed form dynamics do not accurately predict the dynamics for more than perhaps a half or full period. The second issue is that, even if the closed form dynamics were good, the energy metric couples the angle of attack and sideslip equations, such that a closed form solution is not possible. The release time would need to be solved numerically.

However, if numeric approaches are going to be used, it makes more sense to integrate the simplified dynamics developed in Section II.C, and then compute the energy using the integrated state. This was done using the CDT 3-11 BET that was used to validate the simplified dynamics, and the results are plotted in Figure 14. The minimum energy points most likely match better than the maximums because the simplified dynamics match the frequency and phasing of the motion better than the amplitude. In any case, the minimums are the points of interest to an smart drogue release algorithm, and it is clear that the simplified dynamics predict the value and time of the minimums well.

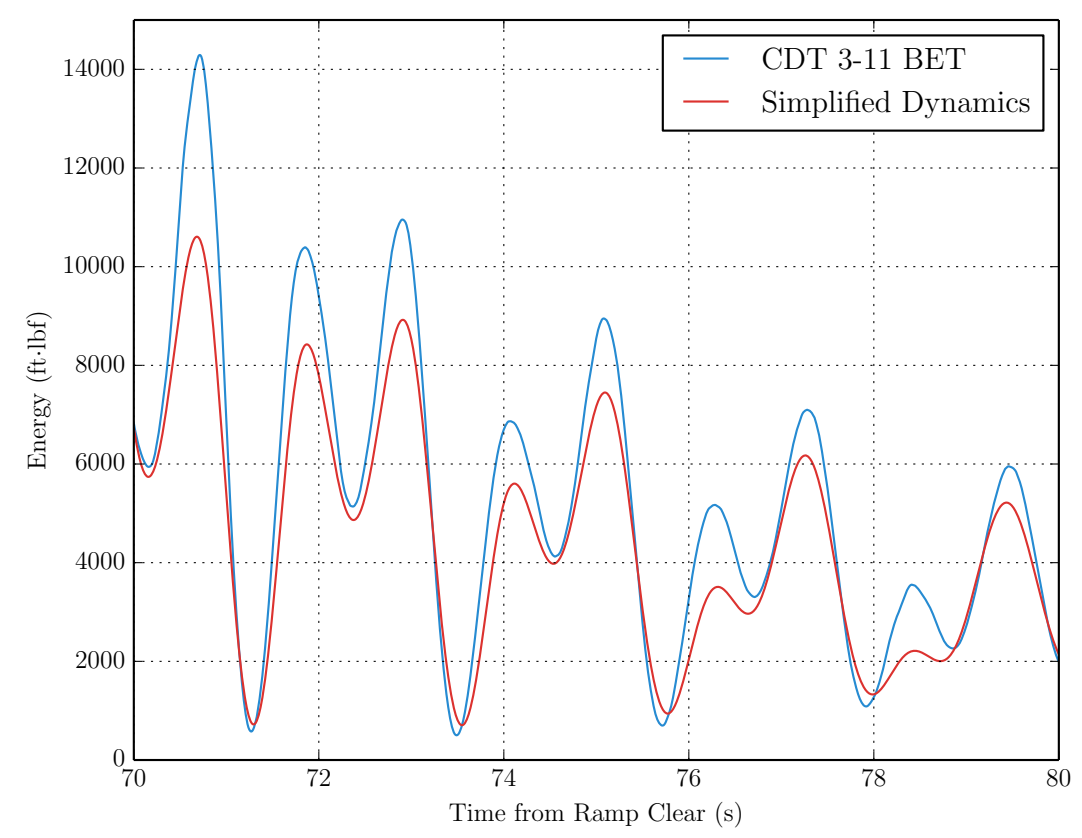

Figure 14. Integrating the simplified dynamics provides a good prediction of the energy metric.

The integrated simplified equations and the energy metric were then combined to create a numeric predictor algorithm. The algorithm integrates the state forward from the current condition to the end of the drogue release window. It computes the energy at each integration step. The time corresponding to the minimum energy in the entire window is selected as the time to go until release. A benefit of working with time as the indepenedent variable is that the estimated drogue release latency can be accounted for directly by biasing the time to go.

The proposed numeric predictor algorithm begins by integrating the current rotational state forward by the drogue release latency time. This gives the physical release state that corresponds to an immediate release $\left(t_{\mathrm{go}}=0 \mathrm{~s}\right)$. The energy is computed at this state and stored. The time is then reset to 0 so that it now corresponds to release command time, while the state corresponds to the physical release state. Then, the state is marched forward, and the energy is computed at each point. If the computed energy is lower than the stored value, the time is taken as the release time. The process ends when the release time reaches the end of the release window. If the time to go is zero, then an immediate release corresponds to the minimum energy in the window, and release is commanded.

An example of this algorithm at work is shown in Figure 15. The drogue release window opens at $45 \mathrm{~s}$ and closes at $50 \mathrm{~s}$. The algorithm at first predicts that the minimum occurring just after $48 \mathrm{~s}$ will be the best in the window, and the time to go counts down to it. However, as the end of the window nears, the predictions improve, and the algorithm realizes that the minimum just before $50 \mathrm{~s}$ is actually better, and it 
switches to that solution. The time to go proceeds to zero, and release is commanded.

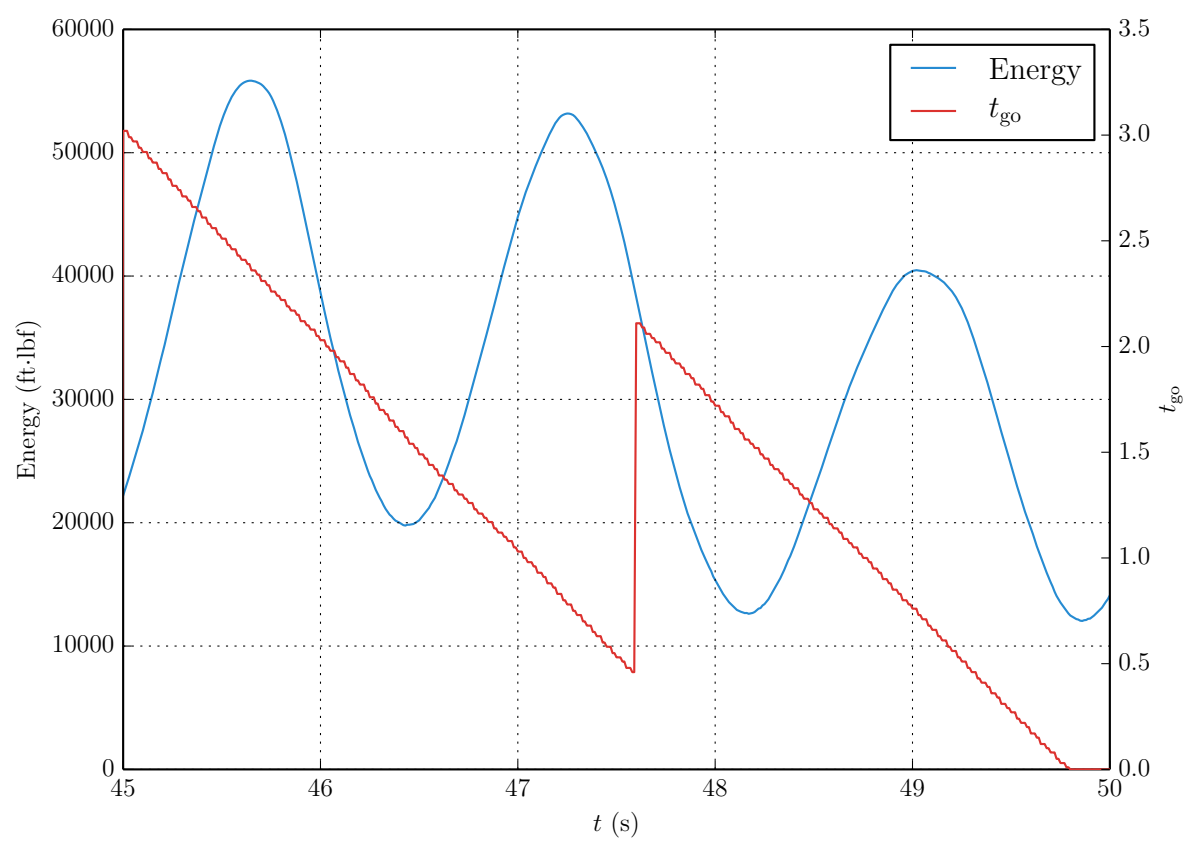

Figure 15. Integrating the simplified dynamics provides a good prediction of the energy metric.

This method has several advantages over its predecessors. First, it is predictive, whereas the older algorithms only compared the current state to the previous oscillation. The older algorithms had no capability to know if a better release condition would occur later in the release window. Second, this algorithm combines the concepts of minimizing the RSS rate and minimizing the angle from free flight trim into a single scalar term. The algorithm faces none of the issues that are created by having two separate conditions that must overlap. Lastly, since it uses time as an independent variable, it can account for the drogue release latency directly, without any need for tuning.

The algorithm is certainly more complex than its predecessors. It requires more input parameters, though they are basic vehicle parameters and not tuning parameters. It also requires more navigated state information. It requires the angle of attack, sideslip angle, angular velocity, dynamic pressure, and freestream velocity magnitude. The algorithm is still much less complex than, for example, an entry guidance algorithm. Whereas a guidance may need to do a line search, this algorithm simply sweeps over time once per call. There is no optimization that needs to converge and no need to integrate the equations of motion multiple times per call.

\section{Performance Comparison}

\section{IV.A. Monte Carlo Results}

The three algorithms were implemented in the Flight Analysis and Simulation Tool (FAST), a 6 degree of freedom simulation used by CPAS for pre-test analysis and post-flight reconstruction. The simulation makes use of the MPCV Hifi Parachute Model, which models each parachute as a separate 6 degree of freedom body, connected to the MPCV with an elastic riser. The simulation uses the Earth Global Reference Atmospheric Model (GRAM) for atmosphere and wind modeling.

A Monte Carlo analysis was performed for each algorithm. The scenario was a drogue failure, which is the case that stresses the baseline algorithm. A perfect navigation model was used. However, the GRAM atmosphere and winds are dispersed, and the navigation model has no knowledge of the wind velocity. The wind speeds are as high as $60 \mathrm{ft} / \mathrm{s}$, which translates into errors in the angle of attack and sideslip angle as high as $20 \mathrm{deg}$, and errors in the dynamic pressure as high as $4 \mathrm{psf}$. These errors only affect the numeric 
predictor, since the baseline and modified baseline algorithms do not require these inputs.

The algorithms were compared by monitoring the minimum total angle of attack each Monte Carlo case reaches between drogue release and the beginning of main first stage inflation. The total angle of attack is the combination of the angle of attack and sideslip angle, and satisfies the following relationship:

$$
\cos \alpha_{\text {total }}=\cos \alpha \cos \beta
$$

The MPCV convention is that a total angle of attack of $180 \mathrm{deg}$ corresponds to heat shield forward flight. The results for the three algorithms are shown in Figure 16. The baseline algorithm resulted in 17 of the 3000 cases going below a total angle of attack of $90 \mathrm{deg}$, the modified baseline in 11, and the numeric predictor in 2. The density function also shows that the modified baseline algorithm shifted the cluster of points closer to $180 \mathrm{deg}$, and the numeric predictor even more so. The attitude at main bag extraction is compared in Figure 17. The modified baseline is slightly skewed towards higher angles of attack, and the numeric predictor more dramatically so. The less extreme attitudes from heat shield forward decrease the likelihood of one of the main parachute bags becoming entrapped in the MPCV's forward bay.

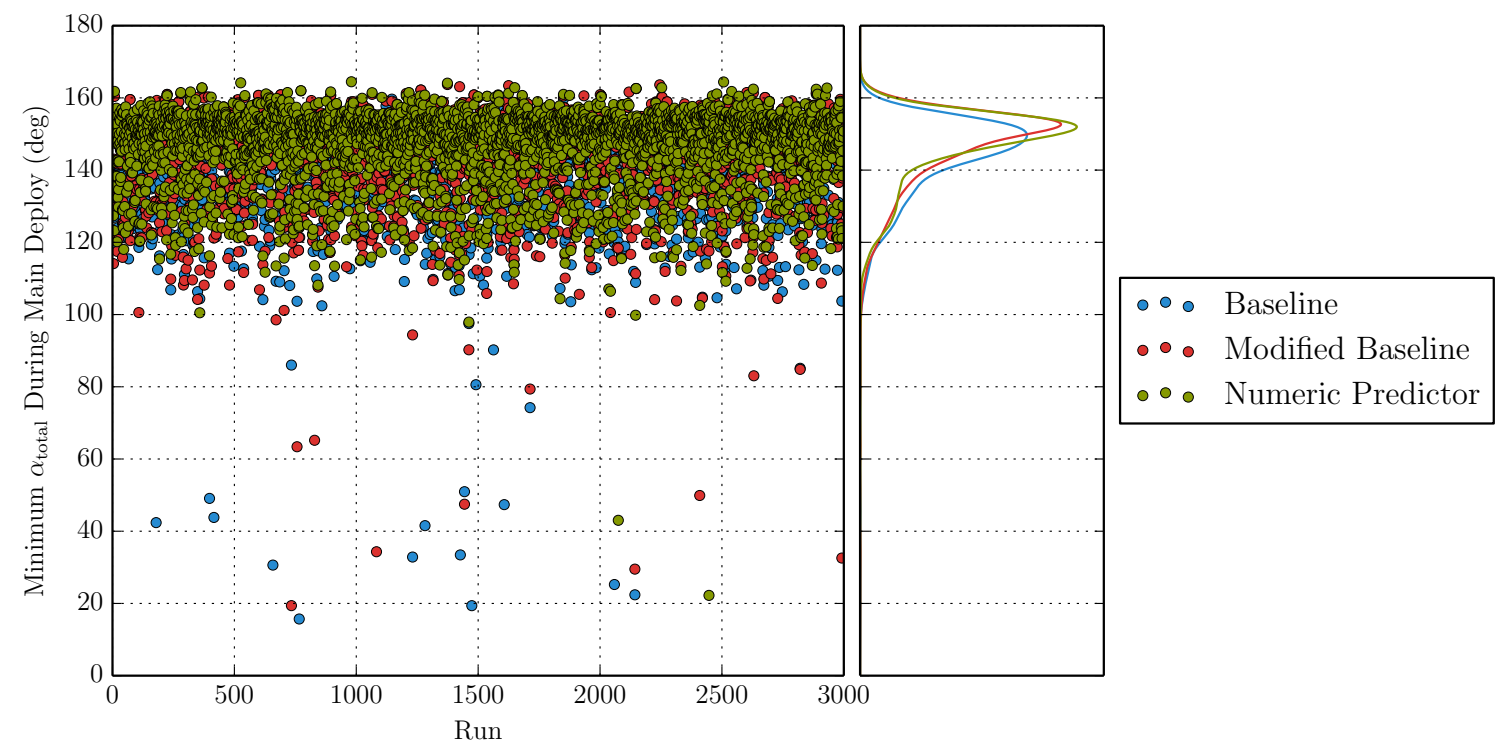

Figure 16. Minimum total angle of attack between drogue release and the beginning of main inflation.

\section{IV.B. Sensitivity to Aerodynamics}

A set of Monte Carlos were run to stress the algorithms with the worst case MPCV dynamic aerodynamics. The Monte Carlo inputs are the same as above, including the drogue failure, but the dispersion on the dynamic aerodynamic coefficients was fixed to the most undamped configuration for every run. This results in even less damping during the time under the drogues, and so higher amplitude attitude oscillations at the end of the drogue ride in more cases. The minimum total angle of attack during main deployment for each algorithm is compared in Figure 18. The baseline algorithm results in 218 cases below a total angle of attack of $90 \mathrm{deg}$, the modified baseline in 122, and the numeric predictor in 6 . The density function shows that the modified baseline keeps more cases towards the higher total angles of attack than the baseline, and the numeric predictor much more so.

\section{IV.C. Sensitivity to Navigation Errors}

The baseline and modified baseline algorithms require only angular velocity data from the navigation system. The angular velocity errors are very small and thus do not impact the performance of these two algorithms.

The numeric predictor, however, also requires angle of attack, sideslip angle, freestream velocity magnitude, and dynamic pressure. These parameters are all derived from the inertial position and velocity. FAST 


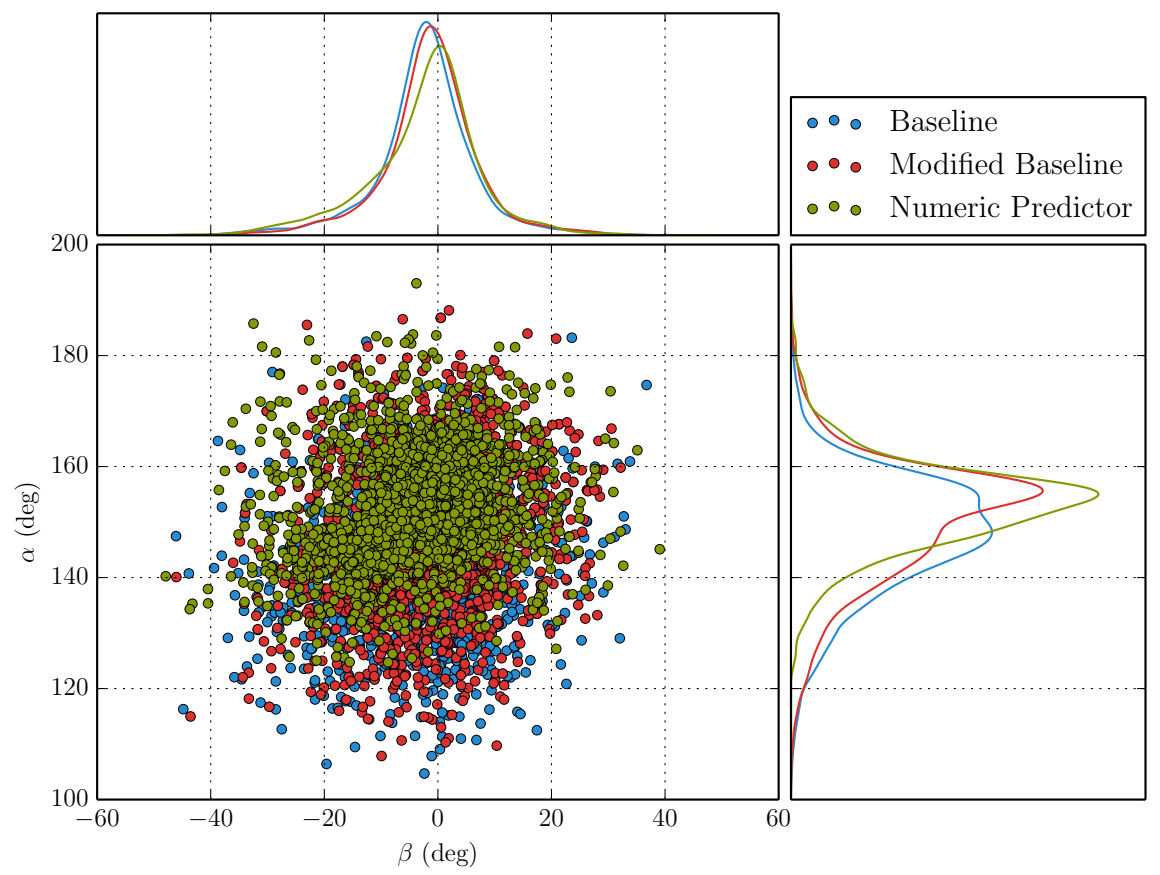

Figure 17. Angle of attack vs. sideslip angle at main bag extraction.

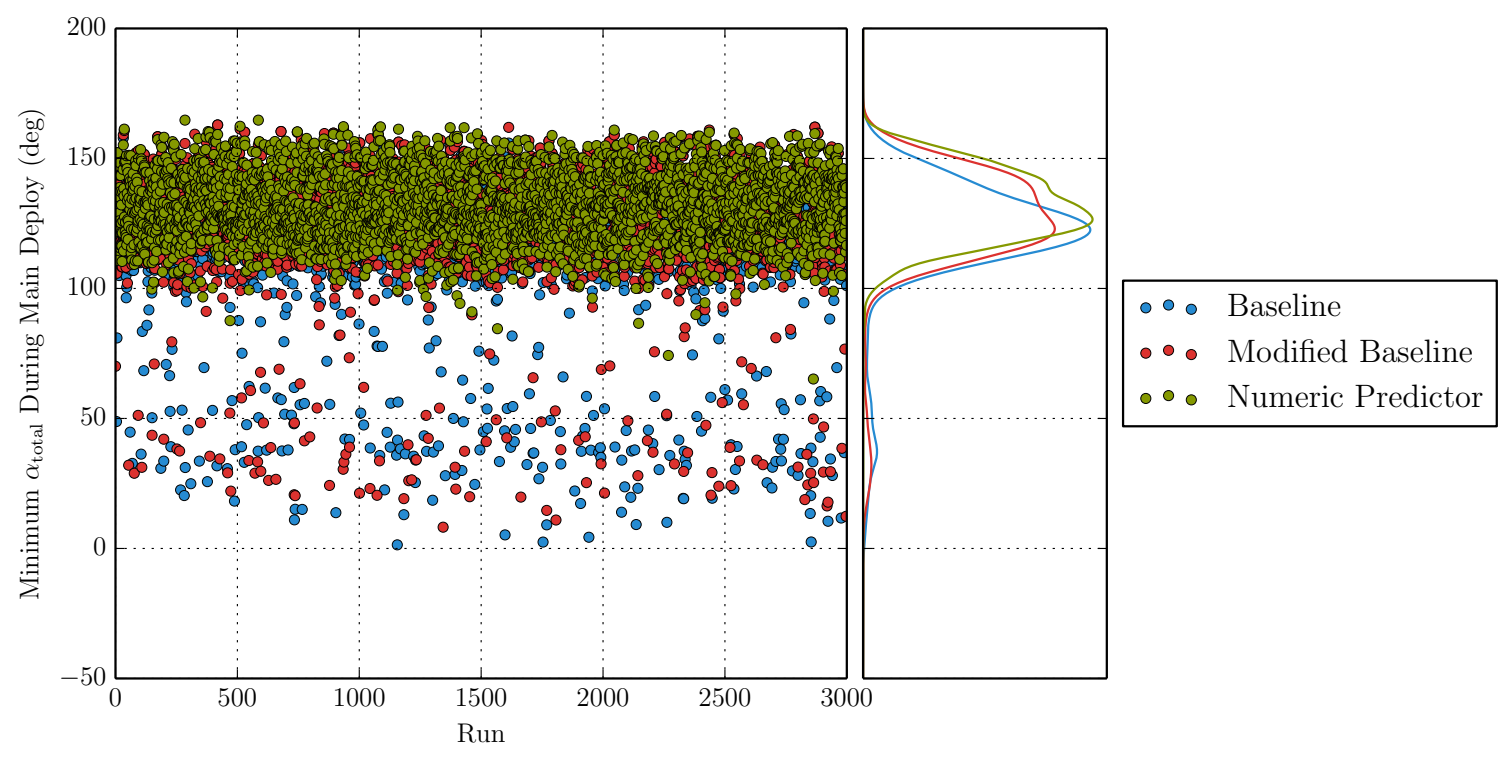

Figure 18. Minimum total angle of attack between drogue release and the beginning of main inflation using the most undamped MPCV dynamic aerodynamics. 
does not have a high fidelity model of the Orion navigation system, but a simple model was implemented to add a constant error term to the truth data. This error term can them be dispersed. These dispersions are applied in addition to the errors caused by the lack of wind knowledge.

A set of Monte Carlos were run using uncorrelated errors representative of a navigation failure scenario where GPS lock was lost just prior to entry interface and never reacquired. For this case, the maximum inertial position error magnitudes were $1500 \mathrm{~m}$ in the $x$ direction, $1500 \mathrm{~m}$ in the $y$ direction, and $1000 \mathrm{~m}$ in the $z$ direction. The maximum inertial velocity error magnitudes were $4 \mathrm{~m} / \mathrm{s}$ in the $x$ direction, $5 \mathrm{~m} / \mathrm{s}$ in the $y$ direction, and $4 \mathrm{~m} / \mathrm{s}$ in the $z$ direction. Since the velocity errors are small compared to the wind speed, there is only a small change in the errors of the derived parameters.

The minimum total angle of attack during main deployment for the loss of GPS case is compared to the earlier perfect navigation case in Figure 19. Again, since the velocity errors are small compared to the wind speed, the performance of the algorithm is more or less unaffected.

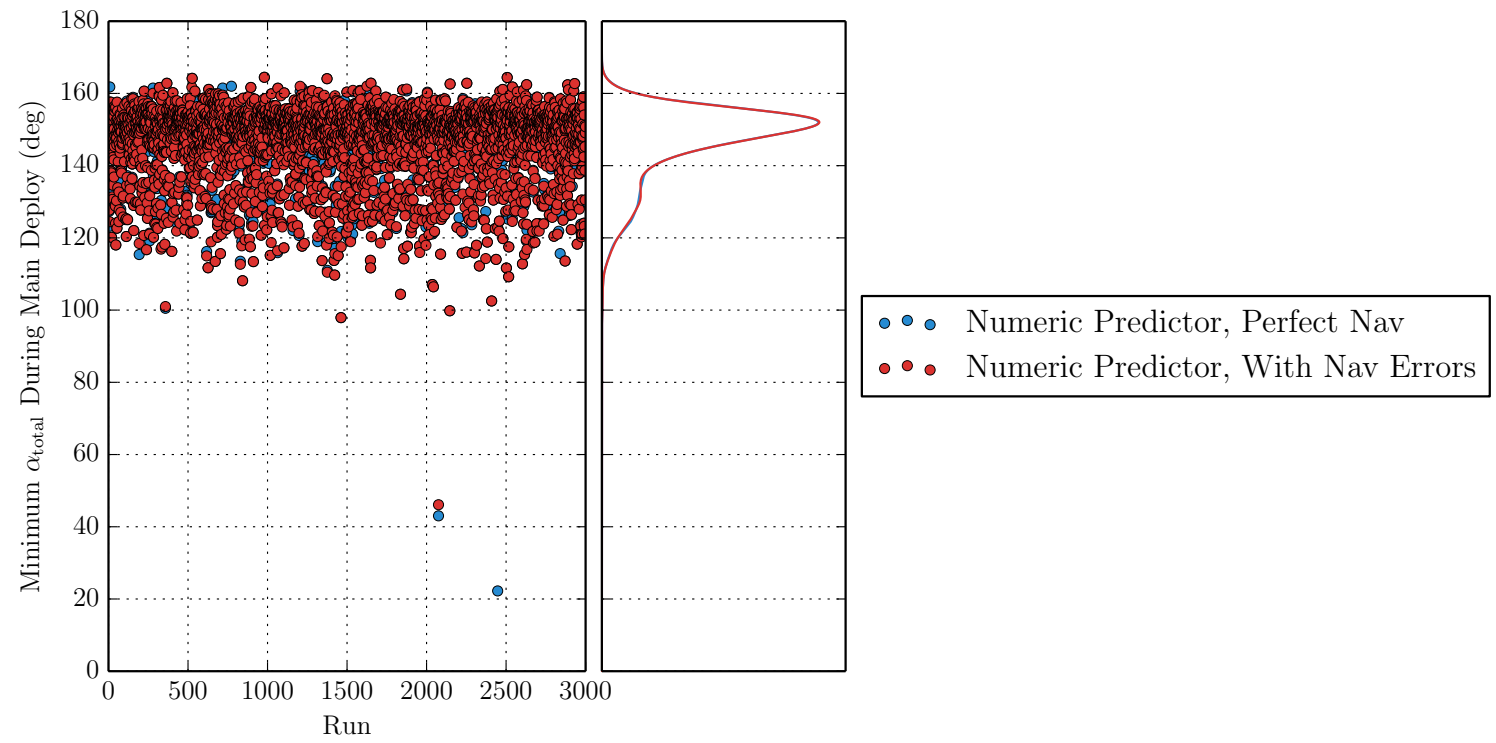

Figure 19. Sensitivity of the numeric predictor performance to errors in the navigated state.

If it is necessary for the algorithm to operate after a more severe navigation system failure, it may be possible to correct much of the error in the navigated angle of attack and sideslip angle. While the wind velocity changes fairly rapidly with time, a navigation error changes much more slowly. An error in the inertial position or velocity would manifest as a shift in the angle of attack and sideslip angle. By comparing the running average of both parameters to their expected values while under the drogues, a correction for each parameter could be determined.

\section{IV.D. Sensitivity to Execution Rate}

The baseline and modified baseline algorithms are simple and do not require much computation time. The numeric predictor, however, must integrate the equations of motion and so requires more floating point operations and thus more computational time. Fortunately, by the time the vehicle reaches the drogue release window, the flight software has a relatively light load, since the entry algorithms are idle.

However, if for some reason the numeric predictor were to prove too computationally expensive, an easy mitigation would be to simply run it at a lower execution rate. The results above all use an execution rate of $40 \mathrm{~Hz}$. This is the rate used by CPAS for the drop test vehicles. Additional Monte Carlo cases were run for execution rates of $10 \mathrm{~Hz}$ and $5 \mathrm{~Hz}$. The Orion entry guidance algorithm, PredGuid, runs at $5 \mathrm{~Hz}$, so this seems a reasonable bound. The minimum total angle of attack during main deployment is compared in Figure 20, and it shows that there is little sensitivity to the execution rate.

If the rate were indeed decreased, the period of time between consecutive executions of the algorithm, and thus opportunities to command release, could become a large percentage of the period of motion under 


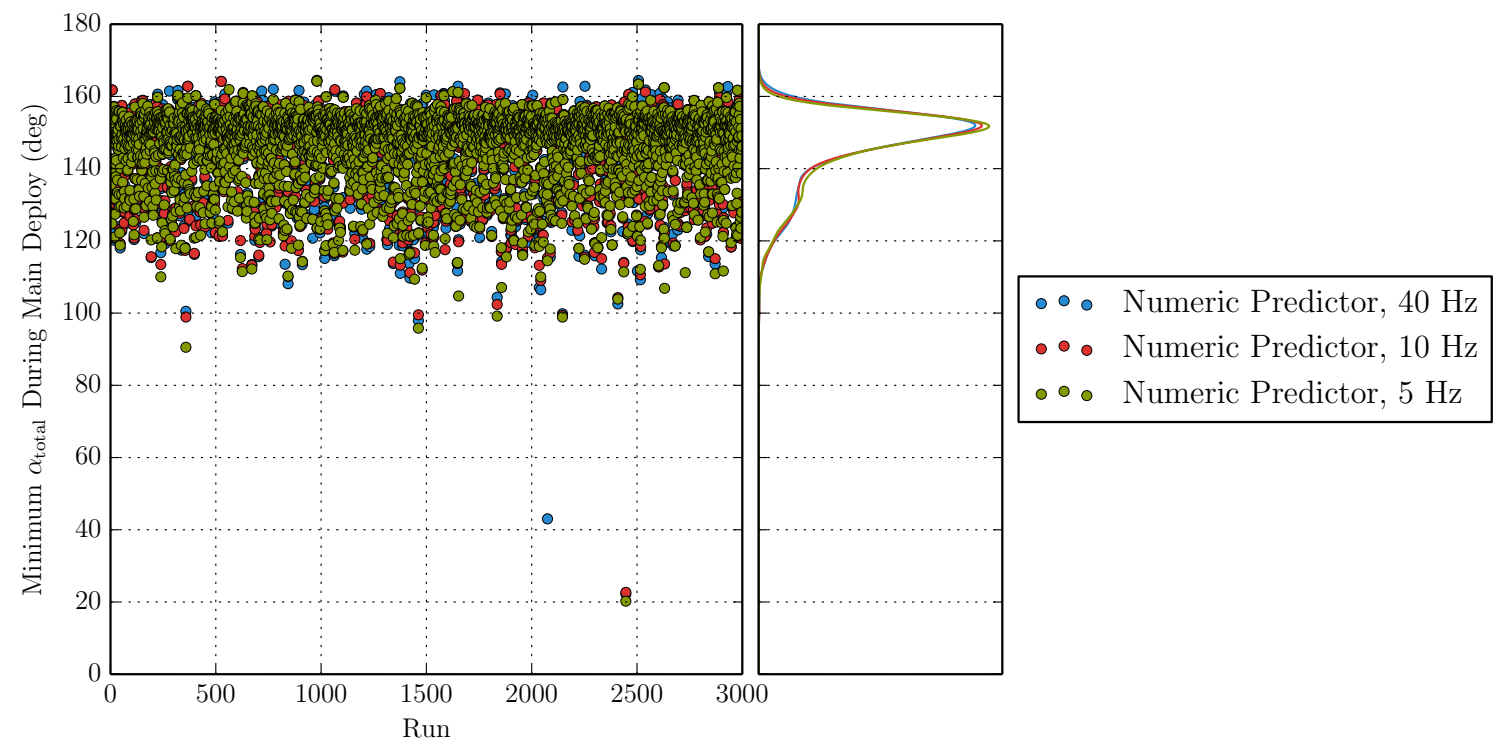

Figure 20. Sensitivity of the numeric predictor performance to execution rate.

the drogues. In this case, it may be necessary to implement a higher rate fine count process to command the release with better resolution once the lower rate algorithm determined the release time was near.

Also, FAST does not account for the computational time of the algorithm itself, only the rate of execution. But the numeric predictor is already accounting for the time delay between the flight software command and physical release. The computational time can also be accounted for this way, by simply adding an estimate of the computational time to the drogue release latency parameter.

\section{Conclusion}

The baseline smart drogue release algorithm does not perform as well as desired in drogue failure cases, where the attitude oscillations at drogue release are large. A modified baseline algorithm was developed to incorporate knowledge of the difference between the hang angle under the drogues and the free flight trim angle, and shows a modest performance improvement in many cases. However, the implementation involves two separate conditions which must overlap, which led to situations where the algorithm may never identify a good release condition. A numeric predictor algorithm was developed, which integrates simplified equations of motion over the release window to select the drogue release time that minimizes a rotational energy metric.

Though it is more complex than the first two algorithms, the numeric predictor offers significant advantages. While the first two algorithms only compare the current state to the most recent oscillation, the numeric predictor integrates forward and can select the best release point in the entire drogue release window. Also, the numeric predictor combines the angular velocity and attitude from trim information into a single scalar energy metric, and thus does not suffer from the same issues as the modified baseline algorithm. Lastly, because the numeric predictor deals with time as the independent variable, it can directly account for the estimated drogue release latency, where the other algorithms rely on a Monte Carlo tuning process.

These advantages resulted in improved performance over both the baseline and modified baseline algorithms. The numeric predictor's performance gains were made even more apparent by looking at a case with the most undamped MPCV aerodynamics. Because of its increased complexity and increased reliance on navigated state, additional cases were run to show that the numeric predictor is relatively insensitive to execution rate and errors in the navigated state. 


\section{Acknowledgments}

Daniel Matz thanks Joe Gamble and Roger Wacker for sharing their thoughts on the drogue release problem and starting this research task off in the right direction.

\section{References}

${ }^{1}$ Machin, Ricardo A., and Evans, Carol T., "Cluster Development Test 2 an Assessment of a Failed Test," 20th AIAA Aerodynamic Decelerator Systems Technology Conference and Seminar, Seattle, WA, 2009.

${ }^{2}$ Matz, Daniel, "Response to PA-1 1.7 Design Cycle iVAC RFA 2-7: Chute Dynamics Analysis," FltDyn-CEV-10-2, 2010.

${ }^{3}$ Stachowiak, Susan J., "Orion Program Pad Abort \#1 Flight Test Flight Dynamics Post-Flight Report Volume 2," FltDynCEV-10-118, 2010.

${ }^{4}$ Currin, Kelly M., Gamble, Joe D., Matz, Daniel A., Bretz, David R., "Evaluation of Drogue Parachute Damping Effects Utilizing the Apollo Legacy Parachute Model," 30th AIAA Applied Aerodynamics Conference, New Orleans, LA, 2012

${ }^{5}$ Aubuchon, Vanessa V., Owens, D. Bruce, Fremaux, C. Michael, "Drogue Parachute Effects on the Orion Crew Module Stability," 29th AIAA Applied Aerodynamics Conference, Honolulu, HI, 2011.

${ }^{6}$ Bledsoe, Kristin J., "Application of a Smart Parachute Release Algorithm to the CPAS Test Architecture," AIAA Aerodynamic Decelerator Systems Conference, Daytona Beach, FL, 2013. 